MATHEMATICS OF COMPUTATION

Volume 72 , Number 241, Pages 105-129

S 0025-5718(02)01411-4

Article electronically published on May 1, 2002

\title{
FULL-WAVE ANALYSIS OF DIELECTRIC WAVEGUIDES AT A GIVEN FREQUENCY
}

\author{
L. VARDAPETYAN AND L. DEMKOWICZ
}

\begin{abstract}
New variational formulation to compute propagation constants is proposed. Based on it, vector finite element method is proved to exclude spurious modes provided finite elements possess discrete compactness property. Convergence analysis is conducted in the framework of collectively compact operators. Reported theoretical results apply to a wide class of vector finite elements including two families of Nedelec and their generalization, the $h p$ edge elements. Numerical experiments fully support theoretical estimates for convergence rates.
\end{abstract}

\section{INTRODUCTION}

Vector finite elements have been successfully used in the analysis and design of electromagnetic resonators. Standard Nedelec elements of [26] have been tested in practice to deliver solutions free of spurious modes. These elements can be readily applied to waveguides for the computation of cutoff frequencies $\omega$ for modes with a given propagation constant $\beta$. The case with a given real-valued $\beta$ has been extensively studied, see for example [7, 17]. In this paper, we are interested in the more physically relevant case, when $\omega$ is given but $\beta$ is unknown. It differs significantly from the well-studied one. In general, even nonlossy waveguides yield nonselfadjoint eigenvalue problems with possibly complex-valued $\beta$ and the corresponding evanescent modes. To the best of the authors' knowledge, this problem has been addressed only by the engineering community (for a review see [31]), with no comprehensive convergence analysis. Moreover, an existing approach to this problem allows for an infinite dimensional subspace of spurious modes corresponding to propagation constant $\beta=0$, see $[23,22]$. These spurious modes may pollute numerical TEM-like solutions, especially as $\omega \rightarrow 0$, if no extra care is taken in the numerical scheme. Our goal has been to develop a variational formulation applicable for quasistatic regimes and to provide a comprehensive mathematical analysis of a suitable finite element discretization with convergence rate estimates. The proposed variational formulation of the appropriate eigenvalue problem (although less memory-efficient than in 23, 22, is uniformly stable as $\omega \rightarrow 0$, does not suffer from spurious modes 1 and, leading to the spectral analysis of a compact operator, greatly simplifies the

Received by the editor January 11, 2000 and, in revised form, February 20, 2001.

2000 Mathematics Subject Classification. Primary 65N30, 35L15.

Key words and phrases. Maxwell's equations, waveguide eigenmodes, full-wave analysis, $h p$ finite elements.

${ }^{1}$ One might argue that now $\beta=\infty$, not $\beta=0$ as in [23] [22], has infinite multiplicity and spurious eigenmodes. However, the fields corresponding to $\beta=\infty$ are completely eliminated from actual computations. 
mathematical analysis. As a practical advantage, zero is not an eigenvalue and all (noninfinite) eigenvalues have finite multiplicities. In this paper, we develop a new formulation, prove it to be free of spurious modes, and study convergence properties of vector finite element methods based on this formulation. The convergence results apply to any finite elements which have discrete compactness and appropriate approximability properties, see 18 . This includes two families of Nedelec elements [26, 27] and their generalization, the $h p$-adaptive elements of [14, 33]. The rates of convergence relate to the interpolation error estimates. We present convergence rates for Nedelec elements [26, 27] and show that numerical experiments confirm the theoretical estimates. We refer to [32] for additional numerical examples.

\section{Problem Setup}

Let us consider a closed waveguide defined by a right cylinder with cross section $\Omega \subset \mathbb{R}^{2}$. The waveguide is filled with inhomogeneous media. Real-valued functions $\epsilon$ and $\mu$ describe the electromagnetic properties of the waveguide. As in [17], we assume that the functions $\epsilon$ and $\mu$ are piecewise Lipschitz continuous and have no variation along the waveguide. $\Omega$ is a bounded, Lipschitz, simply connected polyhedral domain with boundary $\Gamma$.

We are interested in finding solutions to Maxwell's equations which propagate along the source-free waveguide. The general ansatz for such fields is given by

$$
\begin{aligned}
& \mathcal{E}\left(\boldsymbol{x}, x_{3}, t\right)=\left(\boldsymbol{E}(\boldsymbol{x}), E_{3}(\boldsymbol{x})\right) e^{\jmath\left(\omega t \mp \beta x_{3}\right)}, \\
& \mathcal{H}\left(\boldsymbol{x}, x_{3}, t\right)=\left(\boldsymbol{H}(\boldsymbol{x}), H_{3}(\boldsymbol{x})\right) e^{\jmath\left(\omega t \mp \beta x_{3}\right)},
\end{aligned}
$$

where $\boldsymbol{x} \in \Omega$ and the $x_{3}$-axis is along the waveguide. The positive number $\omega$ denotes frequency, and $\beta$ is the constant of propagation. $\boldsymbol{E}$ and $\boldsymbol{H}$ are electric and magnetic field components in the plane of the cross section, and $E_{3}$ and $H_{3}$ are electric and magnetic components along the waveguide. With ansatz (2.1), the second order 3D Maxwell equations expressed in terms of electric field $\left(\boldsymbol{E}, E_{3}\right)$ alone reduce to three $2 \mathrm{D}$ equations:

$$
\left\{\begin{array}{l}
\boldsymbol{\nabla} \times\left(\frac{1}{\mu} \boldsymbol{\nabla} \times \boldsymbol{E}\right)-\omega^{2} \epsilon \boldsymbol{E}+\frac{\beta^{2}}{\mu} \boldsymbol{E}-\frac{\jmath \beta}{\mu} \nabla E_{3}=0, \\
\boldsymbol{\nabla} \circ\left(\frac{1}{\mu} \nabla E_{3}\right)+\omega^{2} \epsilon E_{3}+\jmath \beta \nabla \circ\left(\frac{1}{\mu} \boldsymbol{E}\right)=0, \\
\boldsymbol{\nabla} \circ(\epsilon \boldsymbol{E})-\jmath \beta \epsilon E_{3}=0 .
\end{array}\right.
$$

For simplicity, perfect electric conductor boundary conditions are imposed on $\Gamma$ :

$$
\boldsymbol{E} \times \boldsymbol{n}=0, \quad E_{3}=0,
$$

where $\boldsymbol{n}$ is the outward unit normal on $\Gamma$. Since no sources are given, (2.2) is an eigenvalue problem. Either $\omega$ or $\beta$ is assumed to be known, and the goal is to find all possible pairs which consist of the other missing constant $\beta$ (or $\omega$ ) and the corresponding electric field $\left(\boldsymbol{E}, E_{3}\right)$ that solve (2.2) and satisfy (2.3). The case with a given real-valued $\beta$ has been examined in [7, 17]. We are interested in the more physically relevant case, when $\omega$ is given, but $\beta$ is unknown. 


\section{VARIATIONAL FORMULATION}

As mentioned above, our goal is to find all pairs $\left(\beta,\left(\boldsymbol{E}, E_{3}\right)\right)$ which satisfy equations (2.2) and boundary conditions (2.3) for a given frequency $\omega>0$.

Before we construct our variational formulation, let us develop a functional setting suitable for the problem at hand. Let $\mathcal{H}$ denote the Hilbert space $\boldsymbol{L}_{\epsilon}^{2}(\Omega) \times$ $L_{\epsilon}^{2}(\Omega)$, equipped with the norm

$$
\|(\boldsymbol{E}, p)\|_{\mathcal{H}}=\left(\|\boldsymbol{E}\|_{\epsilon}^{2}+\|p\|_{\epsilon}^{2}\right)^{\frac{1}{2}},
$$

where $\|\cdot\|_{\epsilon}$ is the $\epsilon$-weighted $L^{2}$-norm:

$$
\|p\|_{\epsilon}=\left(\int_{\Omega} \epsilon|p|^{2} d \boldsymbol{x}\right)^{\frac{1}{2}} .
$$

We also introduce the Hilbert space $\boldsymbol{X}=\boldsymbol{W} \times V$, where $\boldsymbol{W}$ and $V$ are Hilbert spaces defined below. $\boldsymbol{W}$ will provide us with vector-valued test and trial functions:

$$
\boldsymbol{W} \stackrel{\text { def }}{=}\left\{\boldsymbol{E} \in \boldsymbol{L}_{\epsilon}^{2}(\Omega): \boldsymbol{\nabla} \times \boldsymbol{E} \in \boldsymbol{L}^{2}(\Omega), \boldsymbol{n} \times \boldsymbol{E}=\mathbf{0} \text { on } \Gamma\right\}
$$

equipped with the norm

$$
\|\boldsymbol{E}\|_{\boldsymbol{W}}=\left(\|\boldsymbol{E}\|_{\epsilon}^{2}+\|\boldsymbol{\nabla} \times \boldsymbol{E}\|^{2}\right)^{\frac{1}{2}} .
$$

And $V$ will provide us with scalar-valued test and trial functions:

$$
V \stackrel{\text { def }}{=}\left\{p \in H^{1}(\Omega): p=0 \text { on } \Gamma\right\}
$$

equipped with the norm

$$
\|p\|_{V}=(\nabla p, \nabla p)_{\epsilon}
$$

The norm on $\boldsymbol{X}$ is simply

$$
\|(\boldsymbol{F}, q)\|_{\boldsymbol{X}}=\left(\|\boldsymbol{F}\|_{\boldsymbol{W}}^{2}+\|q\|_{V}^{2}\right)^{\frac{1}{2}} .
$$

The boundary condition in (3.3) is understood in the sense of the generalized Green formula [16], and the boundary condition in (3.5) in the sense of traces.

Using the density of infinitely differentiable functions of finite support in $\mathcal{H}$ as well as in $\boldsymbol{X}$, we may conclude that $\boldsymbol{X}$ is dense in $\mathcal{H}$.

$\boldsymbol{W}_{0}$ is a subspace of $\boldsymbol{W}$ :

$$
\boldsymbol{W}_{0} \stackrel{\text { def }}{=}\{\boldsymbol{E} \in \boldsymbol{W}: \boldsymbol{\nabla} \times \boldsymbol{E}=\mathbf{0}\} .
$$

We also note that, in the context of our setup, the gradient operator maps $V$ onto $\boldsymbol{W}_{0}$ :

$$
\nabla V=\boldsymbol{W}_{0} .
$$

If we multiply $(2.2)_{1}$ by a vector test function $\boldsymbol{F}$, and $(2.2)_{2}$ and $(2.2)_{3}$ by a scalar test function $q$, integrate by parts and apply proper boundary conditions, we can recast initial system (2.2) into the following variational form:

Find pairs $\left(\beta,\left(\boldsymbol{E}, E_{3}\right)\right) \in(\mathbb{C}, \boldsymbol{X})$ such that $\forall(\boldsymbol{F}, q) \in \boldsymbol{X}$ :

$$
\left\{\begin{array}{l}
\left(\frac{1}{\mu} \nabla \times \boldsymbol{E}, \nabla \times \boldsymbol{F}\right)-\omega^{2}(\epsilon \boldsymbol{E}, \boldsymbol{F})+\beta^{2}\left(\frac{1}{\mu} \boldsymbol{E}, \boldsymbol{F}\right)-\jmath \beta\left(\frac{1}{\mu} \nabla E_{3}, \boldsymbol{F}\right)=0, \\
-\left(\frac{1}{\mu} \nabla E_{3}, \nabla q\right)+\omega^{2}\left(\epsilon E_{3}, q\right)-\jmath \beta\left(\frac{1}{\mu} \boldsymbol{E}, \nabla q\right)=0, \\
-(\epsilon \boldsymbol{E}, \nabla q)-\jmath \beta\left(\epsilon E_{3}, q\right)=0 .
\end{array}\right.
$$


First, we consider the special case when $\beta=0$. The problem (3.10) then splits into two problems:

For a given $\omega \in R$, find $\boldsymbol{E} \in \boldsymbol{W}$ such that $\forall(\boldsymbol{F}, q) \in \boldsymbol{W} \times V$

$$
\left\{\begin{array}{l}
\left(\frac{1}{\mu} \nabla \times \boldsymbol{E}, \nabla \times \boldsymbol{F}\right)-\omega^{2}(\epsilon \boldsymbol{E}, \boldsymbol{F})=0, \\
(\epsilon \boldsymbol{E}, \nabla q)=0 ;
\end{array}\right.
$$

For a given $\omega \in R$, find $E_{3} \in V$ such that $\forall q \in V$

$$
\left(\frac{1}{\mu} \nabla E_{3}, \nabla q\right)-\omega^{2}\left(\epsilon E_{3}, q\right)=0 .
$$

Solutions of (3.11) and (3.12) taken as a pair satisfy the original system.

Now let us recall two theorems which play important roles in our analysis. Details can be found in [24].

Theorem 1. With our assumptions on the domain, the spectrum of the following variational eigenvalue problem for the curl-curl operator:

$$
\left\{\begin{array}{l}
\boldsymbol{E} \in \boldsymbol{W}, \nu \in \mathbb{C}, \\
\int_{\Omega} \frac{1}{\mu}(\nabla \times \boldsymbol{E}) \circ(\nabla \times \overline{\boldsymbol{F}}) d \boldsymbol{x}=\nu \int_{\Omega} \epsilon \boldsymbol{E} \circ \overline{\boldsymbol{F}} d \boldsymbol{x} \quad \forall \boldsymbol{F} \in \boldsymbol{W}, \\
\int_{\Omega} \epsilon \boldsymbol{E} \circ \nabla \bar{q} d \boldsymbol{x}=0 \quad \forall q \in V,
\end{array}\right.
$$

is a pure point spectrum on the positive real axis, extending to infinity:

$$
\left\{0<\nu_{1} \leq \nu_{2} \leq \cdots \leq \nu_{n} \leq \cdots<+\infty\right\} .
$$

Theorem 2. With our assumptions on the domain, the spectrum of the following variational eigenvalue problem for the div-grad operator:

$$
\left\{\begin{array}{l}
p \in V, \eta \in \mathbb{C}, \\
\int_{\Omega} \frac{1}{\mu}(\nabla p) \circ(\nabla \bar{q}) d \boldsymbol{x}=\eta \int_{\Omega} \epsilon p \bar{q} d \boldsymbol{x} \quad \forall q \in V,
\end{array}\right.
$$

is a pure point spectrum on the positive real axis, extending to infinity:

$$
\left\{0<\eta_{1} \leq \eta_{2} \leq \cdots \leq \eta_{n} \leq \cdots<+\infty\right\} .
$$

It follows from the theorems above that $\beta=0$ has a nontrivial eigenmode $\left(\boldsymbol{E}, E_{3}\right)$ associated with it if and only if $\omega^{2}$ is an eigenvalue of (3.13) or (3.14). Thus, the multiplicity of $\beta=0$ is equal to the sum of multiplicities of $\omega^{2}$ that is an eigenvalue of (3.13) or (3.14). Therefore, zero as an eigenvalue of (3.10) may have only a finite multiplicity.

Let us now narrow our task and seek only those solutions of (3.10) that correspond to a nonzero propagating constant $\beta$. In other words, we assume that

$$
\text { - } \boldsymbol{R}(\omega) \text { : Frequency } \omega \notin\left\{\eta_{i}\right\}_{i=1}^{\infty} \cup\left\{\nu_{j}\right\}_{j=1}^{\infty} \text {. }
$$

Clearly, all propagating modes are among these solutions. We note that the constant $\beta$ enters system (3.10) in both the first and second powers. However, if the 
component $E_{3}$ is rescaled as $E_{3}^{\text {new }}=\jmath \beta E_{3}$ or $E_{3}^{\text {new }}=\frac{1}{\jmath \beta} E_{3}$, then only $\beta^{2}$ is present in the modified system.

Moreover, since $\beta \neq 0$, the equations in (3.10) are not all linearly independent. Indeed, if $\boldsymbol{F}=\nabla q$, then $(3.10) 1$ yields

$$
-\omega^{2}(\epsilon \boldsymbol{E}, \nabla q)+\beta^{2}\left(\frac{1}{\mu} \boldsymbol{E}, \nabla q\right)-\jmath \beta\left(\frac{1}{\mu} \nabla E_{3}, \nabla q\right)=0 .
$$

Now we can express the first term of (3.16) via $\left(\epsilon E_{3}, q\right)$ of $(3.10) 3$ to get

$$
\jmath \beta\left[\omega^{2}\left(\epsilon E_{3}, q\right)-\jmath \beta\left(\frac{1}{\mu} \boldsymbol{E}, \nabla q\right)-\left(\frac{1}{\mu} \nabla E_{3}, \nabla q\right)\right]=0,
$$

which yields $(3.10){ }_{2}$ multiplied by $\jmath \beta$. Similarly, the difference of $(3.10)_{2}$ multiplied by $\jmath \beta$ and (3.16) gives (3.10) 3 multiplied by $\omega^{2}$.

Thus, we can actually consider not all three but only two equations of the variational system (3.10). The question is: which equation to discard? And how to rescale $E_{3}$ ? Following the logic of our previous papers [14] and [33], we keep $(3.10)_{3}$, which explicitly involves a weak divergence condition on $\boldsymbol{E}$, and turn it into a constraint on $\left(\boldsymbol{E}, E_{3}\right)$ by rescaling $E_{3}$ as follows:

$$
E_{3}^{\text {new }}=\jmath \beta E_{3} .
$$

To limit our notation, $E_{3}$ will represent $E_{3}^{\text {new }}$ for the remainder of this paper.

With the proposed simplifications, formulation (3.10) reduces to:

For a given $\omega>0$, find all pairs $\left(\beta,\left(\boldsymbol{E}, E_{3}\right)\right) \in \mathbb{C} \times \boldsymbol{X}, \quad \beta \neq 0$, such that $\forall(\boldsymbol{F}, q) \in \boldsymbol{X}$

$$
\left\{\begin{array}{l}
\left(\frac{1}{\mu} \nabla \times \boldsymbol{E}, \nabla \times \boldsymbol{F}\right)-\omega^{2}(\epsilon \boldsymbol{E}, \boldsymbol{F})-\left(\frac{1}{\mu} \nabla E_{3}, \boldsymbol{F}\right)=-\beta^{2}\left(\frac{1}{\mu} \boldsymbol{E}, \boldsymbol{F}\right), \\
-(\epsilon \boldsymbol{E}, \nabla q)-\left(\epsilon E_{3}, q\right)=0 .
\end{array}\right.
$$

We stress that for nonzero $\beta$ the new formulation is equivalent to the original one. Later on, we will show that the new formulation does not allow nontrivial solutions if $\beta=0$.

Remark 1. In 23, 22, Lee et al. kept (3.10) $)_{1}$ and $(3.10)_{2}$ and used a transformation equivalent to $E_{3}=\jmath \beta E_{3}^{\text {new }}$ to get a symmetric system. When discretized, this formulation leads to a generalized eigenvalue problem with a singular matrix, whose spurious solutions that correspond to $\beta=0$ can present considerable complications, especially at low frequencies.

\section{Analysis of the CONTINUOUS PROBlem}

In order to discuss the properties of the problem at hand, let us consider a sesquilinear form $\boldsymbol{a}_{\omega}$ defined on $\boldsymbol{X} \times \boldsymbol{X}$ corresponding to the left hand side of (3.19):

$$
\begin{aligned}
& \boldsymbol{a}_{\omega}: \boldsymbol{X} \times \boldsymbol{X} \longrightarrow \mathbb{C} \\
& \boldsymbol{a}_{\omega}\left(\left(\boldsymbol{E}, E_{3}\right),(\boldsymbol{F}, q)\right)=\left(\frac{1}{\mu} \nabla \times \boldsymbol{E}, \nabla \times \boldsymbol{F}\right)-\omega^{2}(\epsilon \boldsymbol{E}, \boldsymbol{F})-\left(\frac{1}{\mu} \nabla E_{3}, \boldsymbol{F}\right) \\
& +\omega^{2}\left((\epsilon \boldsymbol{E}, \nabla q)+\left(\epsilon E_{3}, q\right)\right) .
\end{aligned}
$$

Clearly, the form $\boldsymbol{a}_{\omega}$ is bounded, i.e., there exists $C>0$ such that

$$
\left|\boldsymbol{a}_{\omega}\left(\left(\boldsymbol{E}, E_{3}\right),(\boldsymbol{F}, q)\right)\right| \leq C\left\|\left(\boldsymbol{E}, E_{3}\right)\right\|_{\boldsymbol{X}}\|(\boldsymbol{F}, q)\|_{\boldsymbol{X}} \quad \forall\left(\boldsymbol{E}, E_{3}\right),(\boldsymbol{F}, q) \in \boldsymbol{X} .
$$


If we also consider the bounded symmetric projection operator $\mathbb{B}: \boldsymbol{X} \longrightarrow \boldsymbol{X}$ defined as

$$
\mathbb{B}(\boldsymbol{F}, q)=\left(\frac{-1}{\epsilon \mu} \boldsymbol{F}, 0\right),
$$

then, with the new notation, (3.19) becomes:

For a given $\omega>0$, find all pairs $\left(\beta,\left(\boldsymbol{E}, E_{3}\right)\right) \in \mathbb{C} \times \boldsymbol{X}, \quad \beta \neq 0$, such that $\forall(\boldsymbol{F}, q) \in \boldsymbol{X}$

$$
\boldsymbol{a}_{\omega}\left(\left(\boldsymbol{E}, E_{3}\right),(\boldsymbol{F}, q)\right)=\beta^{2}\left(\mathbb{B}\left(\boldsymbol{E}, E_{3}\right),(\boldsymbol{F}, q)\right)_{\epsilon} .
$$

We show now that, with the assumption $\boldsymbol{R}(\omega)$ on $\omega$, the linear operator $L$ : $\boldsymbol{X} \longrightarrow \boldsymbol{X}^{\prime}$ defined by the form $a_{\omega}$,

$$
\left\langle L\left(\boldsymbol{E}, E_{3}\right),(\boldsymbol{F}, q)\right\rangle:=\boldsymbol{a}_{\omega}\left(\left(\boldsymbol{E}, E_{3}\right),(\boldsymbol{F}, q)\right) \quad \forall(\boldsymbol{F}, q) \in \boldsymbol{X}
$$

is an isomorphism. First, it follows from (4.2) that $L$ is a continuous operator:

$$
\|L\|_{\mathcal{L}\left(\boldsymbol{X}, \boldsymbol{X}^{\prime}\right)} \leq C .
$$

Now, let us confirm that for any $(\boldsymbol{J}, Q) \in \boldsymbol{X}^{\prime}$ there exists a unique pair $\left(\boldsymbol{E}, E_{3}\right) \in \boldsymbol{X}$ such that

$$
\left.\boldsymbol{a}_{\omega}\left(\boldsymbol{E}, E_{3}\right),(\boldsymbol{F}, q)\right)=\langle(\boldsymbol{J}, Q),(\boldsymbol{F}, q)\rangle, \quad \forall(\boldsymbol{F}, q) \in \boldsymbol{X} .
$$

The explicit form of the system (4.7) follows:

Find $\left(\boldsymbol{E}, E_{3}\right) \in \boldsymbol{X}$, such that $\forall(\boldsymbol{F}, q) \in \boldsymbol{X}$ :

$$
\left\{\begin{array}{l}
\left(\frac{1}{\mu} \nabla \times \boldsymbol{E}, \nabla \times \boldsymbol{F}\right)-\omega^{2}(\epsilon \boldsymbol{E}, \boldsymbol{F})-\left(\frac{1}{\mu} \nabla E_{3}, \boldsymbol{F}\right)=\langle\boldsymbol{J}, \boldsymbol{F}\rangle_{1}, \\
\omega^{2}\left[(\epsilon \boldsymbol{E}, \nabla q)+\left(\epsilon E_{3}, q\right)\right]=\langle Q, q\rangle_{2}
\end{array}\right.
$$

here $\langle\cdot, \cdot\rangle_{1}$ denotes the duality pairing on $\boldsymbol{W}$ and $\langle\cdot, \cdot\rangle_{2}$ is the duality pairing on $V$. We solve the above system in two steps. First, we solve for $E_{3}$ and then for $\boldsymbol{E}$ with $E_{3}$ shifted to the source side.

Let us consider (4.8) 1 with $F=\nabla q$ and add that equation to (4.8) 2 :

$$
-\left(\frac{1}{\mu} \nabla E_{3}, \nabla q\right)+\omega^{2}\left(\epsilon E_{3}, q\right)=\langle Q, q\rangle_{2}+\langle\boldsymbol{J}, \nabla q\rangle_{1}, \quad \forall q \in V .
$$

Since $\boldsymbol{J} \in \boldsymbol{W}^{\prime}$,

$$
\left|\langle\boldsymbol{J}, \boldsymbol{\nabla} q\rangle_{1}\right| \leq\|\boldsymbol{J}\|_{\boldsymbol{W}^{\prime}}\|\boldsymbol{\nabla} q\|_{\boldsymbol{W}}=\|\boldsymbol{J}\|_{\boldsymbol{W}^{\prime}}\|q\|_{V}, \quad \forall q \in V .
$$

Thus, we can define $\hat{J} \in V^{\prime}$ by

$$
\langle\hat{J}, q\rangle_{2}:=\langle\boldsymbol{J}, \nabla q\rangle_{1}, \quad \forall q \in V .
$$

It immediately follows from (4.10) that

$$
\|\hat{J}\|_{V^{\prime}} \leq\|\boldsymbol{J}\|_{\boldsymbol{W}^{\prime}}
$$

Thus, $(\hat{J}+Q) \in V^{\prime}$ acts as the source in (4.9):

$$
-\left(\frac{1}{\mu} \nabla E_{3}, \nabla q\right)+\omega^{2}\left(\epsilon E_{3}, q\right)=\langle\hat{J}+Q, q\rangle_{1}, \quad \forall q \in V .
$$


Since $\omega^{2}$ is not an eigenvalue of $\nabla \frac{1}{\mu} \nabla$, a unique solution to (4.13) exists and is bounded in $V$ by $\|\hat{J}+Q\|_{V^{\prime}}$. Utilizing (4.12), we get that

$$
\left\|E_{3}\right\|_{V} \leq \frac{C_{1}}{\gamma_{1}}\left(\|Q\|_{V^{\prime}}^{2}+\|\boldsymbol{J}\|_{W^{\prime}}^{2}\right)^{\frac{1}{2}}
$$

where $C_{1}>0$ is some constant and $\gamma_{1}$ is the stability constant for the Helmholtz equation expressed via the eigenvalues of the operator $\nabla \frac{1}{\mu} \nabla$ as (see [11])

$$
\gamma_{1}=\min _{i=1 \ldots \infty}\left\{\frac{\left|\eta_{i}-\omega^{2}\right|}{\eta_{i}}\right\}
$$

Now, let us use (4.8) to solve for $\boldsymbol{E}$. We move the terms with $E_{3}$ to the right-hand side:

$$
\left\{\begin{array}{l}
\left(\frac{1}{\mu} \nabla \times \boldsymbol{E}, \boldsymbol{\nabla} \times \boldsymbol{F}\right)-\omega^{2}(\epsilon \boldsymbol{E}, \boldsymbol{F})=\left(\frac{1}{\mu} \nabla E_{3}, \boldsymbol{F}\right)+\langle\boldsymbol{J}, \boldsymbol{F}\rangle_{1} \quad \forall \boldsymbol{F} \in \boldsymbol{W} \\
\omega^{2}(\epsilon \boldsymbol{E}, \nabla q)=-\omega^{2}\left(\epsilon E_{3}, q\right)+\langle Q, q\rangle_{2} \quad \forall q \in V
\end{array}\right.
$$

We notice that since $\omega$ is positive, equation $(4.16)_{2}$ is redundant. Indeed, let us use $\boldsymbol{F}=\nabla q$ in (4.16) 1 . And since $E_{3}$ solves (4.9), we can further simplify (4.16) $)_{1}$ to get

$$
\omega^{2}(\epsilon \boldsymbol{E}, \nabla q)+\omega^{2}\left(\epsilon E_{3}, q\right)-\langle Q, q\rangle_{2}=0 .
$$

Therefore equation $(4.16)_{2}$ acts as a constraint. In order to resolve it, we introduce in (4.16) 1 a Lagrange multiplier $p \in V$. After dividing both sides of $(4.16)_{2}$ by $\omega^{2}$ we have

$$
\left\{\begin{array}{c}
\left(\frac{1}{\mu} \nabla \times \boldsymbol{E}, \nabla \times \boldsymbol{F}\right)-\omega^{2}(\epsilon \boldsymbol{E}, \boldsymbol{F})-(\epsilon \nabla p, \boldsymbol{F}) \\
=\left(\frac{1}{\mu} \nabla E_{3}, \boldsymbol{F}\right)+\langle\boldsymbol{J}, \boldsymbol{F}\rangle_{1}, \quad \forall \boldsymbol{F} \in \boldsymbol{W}, \\
(\epsilon \boldsymbol{E}, \nabla q)=-\left(\epsilon E_{3}, q\right)+\left\langle\frac{Q}{\omega^{2}}, q\right\rangle_{2}, \quad \forall q \in V .
\end{array}\right.
$$

Formulations (4.16) and (4.18) are equivalent. Indeed, using (4.9) we can reduce a linear combination of (4.18) 1 with $\boldsymbol{F}=\nabla p$ and (4.18) 2 with $q=p$ to give

$$
(\epsilon \nabla p, \nabla p)=0 \text {. }
$$

Therefore, as an element of $V, p=0$. So, we can solve (4.18) instead of (4.16).

We also notice that, by the Cauchy-Schwarz and Poincaré inequalities,

$$
\left|\left(\epsilon E_{3}, q\right)\right| \leq C\left\|E_{3}\right\|_{\epsilon}\|q\|_{V},
$$

for some $C>0$. Therefore we can define $\hat{E}_{3} \in V^{\prime}$ by

$$
\left\langle\hat{E}_{3}, q\right\rangle_{2}=\left(\epsilon E_{3}, q\right) \quad \forall q \in V .
$$

And by the Poincaré inequality, it follows from (4.20) that

$$
\left\|\hat{E}_{3}\right\|_{V^{\prime}} \leq C\left\|E_{3}\right\|_{V} \text {. }
$$

Similarly, using the Cauchy-Schwarz inequality and the natural assumption that $\epsilon$ and $\mu$ are strictly positive and bounded on $\Omega$, we can find some $C_{2}>0$ so that

$$
\left|\left(\frac{1}{\mu} \nabla E_{3}, \boldsymbol{F}\right)\right| \leq C_{2}\left\|E_{3}\right\|_{V}\|\boldsymbol{F}\|_{W} .
$$


Thus we can define $\boldsymbol{G} \in \boldsymbol{W}^{\prime}$ by

$$
\langle\boldsymbol{G}, \boldsymbol{F}\rangle_{1}=\left(\frac{1}{\mu} \nabla E_{3}, \boldsymbol{F}\right) \quad \forall \boldsymbol{F} \in \boldsymbol{W} .
$$

It follows from (4.23) that

$$
\|\boldsymbol{G}\|_{W^{\prime}} \leq C_{2}\left\|E_{3}\right\|_{V} .
$$

With the newly introduced $\hat{E}_{3}$ and $\boldsymbol{G}$ system (4.18) reads as follows:

$$
\left\{\begin{array}{l}
\left(\frac{1}{\mu} \nabla \times \boldsymbol{E}, \nabla \times \boldsymbol{F}\right)-\omega^{2}(\epsilon \boldsymbol{E}, \boldsymbol{F})-(\epsilon \nabla p, \boldsymbol{F})=\langle\boldsymbol{G}+\boldsymbol{J}, \boldsymbol{F}\rangle_{1}, \quad \forall \boldsymbol{F} \in \boldsymbol{W}, \\
(\epsilon \boldsymbol{E}, \nabla q)=\left\langle\left(\frac{Q}{\omega^{2}}-\hat{E}_{3}\right), q\right\rangle_{2}, \quad \forall q \in V .
\end{array}\right.
$$

Since $\omega^{2}$ is not in the spectrum of $\nabla \times \frac{1}{\mu} \nabla \times$, the bounded sesquilinear form associated with the system (4.26) satisfies the inf - sup conditions; see for example [14] and [33], where the stability constant is computed. Therefore, a unique solution to this system exists and is bounded by $\left\|(\boldsymbol{G}+\boldsymbol{J}),\left(\frac{Q}{\omega^{2}}-\hat{E}_{3}\right)\right\|_{\boldsymbol{X}^{\prime}}$. Using the estimates for $E_{3}, \hat{E}_{3}$, and $\boldsymbol{G}$ in (4.14), (4.22), (4.25), the triangle inequality, and some elementary algebra, we can get an estimate on $\boldsymbol{E}$ :

$$
\|\boldsymbol{E}\|_{\boldsymbol{W}} \leq \frac{C_{3}}{\gamma_{1} \gamma_{2}}\left(\|\boldsymbol{J}\|_{\boldsymbol{W}^{\prime}}^{2}+\|Q\|_{V^{\prime}}^{2}\right)^{\frac{1}{2}} .
$$

Here $C_{3}>0$ is a constant, $\gamma_{1}$ is as in (4.15) and $\gamma_{2}$ is a stability constant, 14]:

$$
\gamma_{2}=\min \left\{\frac{1}{1+\omega^{2}}, \min _{j=1 \ldots \infty}\left\{\frac{\left|\nu_{j}-\omega^{2}\right|}{\nu_{j}}\right\}\right\} .
$$

Therefore we have demonstrated that the continuous operator $L$ is bijective, and the estimates in (4.14) and (4.27) show that $L^{-1}$, its inverse, is bounded. So, by definition, $L$ is an isomorphism, which in turn, means that form $\boldsymbol{a}_{\omega}$ satisfies the inf - sup conditions, see e.g., 5, 28. We formulate our findings in a theorem.

Theorem 3. With the assumption on $\omega$ as in (3.15), the continuous form $\boldsymbol{a}_{\omega}$ satisfies the following conditions:

There exists $C>0$ such that

$$
\sup _{(\boldsymbol{F}, q) \in \boldsymbol{X}} \frac{\left|\boldsymbol{a}_{\omega}\left(\left(\boldsymbol{E}, E_{3}\right),(\boldsymbol{F}, q)\right)\right|}{\|(\boldsymbol{F}, q)\|_{\boldsymbol{X}}} \geq C \gamma_{1} \gamma_{2}\left\|\left(\boldsymbol{E}, E_{3}\right)\right\|_{\boldsymbol{X}} \quad \forall\left(\boldsymbol{E}, E_{3}\right) \in \boldsymbol{X}
$$

and

$$
\begin{aligned}
& \forall(\boldsymbol{F}, q) \in \boldsymbol{X}, \text { such that }(\boldsymbol{F}, q) \neq 0, \\
& \sup _{\left(\boldsymbol{E}, E_{3}\right) \in \boldsymbol{X}}\left|\boldsymbol{a}_{\omega}\left(\left(\boldsymbol{E}, E_{3}\right),(\boldsymbol{F}, q)\right)\right|>0 .
\end{aligned}
$$

The constant in (4.29), $\alpha:=C \gamma_{1} \gamma_{2}$, is referred to as the inf - sup constant. We note that the following conditions are automatically satisfied, see [5]:

$$
\sup _{\left(\boldsymbol{E}, E_{3}\right) \in \boldsymbol{X}} \frac{\left|\boldsymbol{a}_{\omega}\left(\left(\boldsymbol{E}, E_{3}\right),(\boldsymbol{F}, q)\right)\right|}{\|(\boldsymbol{F}, q)\|_{\boldsymbol{X}}} \geq \alpha\left\|\left(\boldsymbol{E}, E_{3}\right)\right\|_{\boldsymbol{X}} \quad \forall(\boldsymbol{F}, q) \in \boldsymbol{X}
$$


and

$$
\begin{aligned}
& \forall\left(\boldsymbol{E}, E_{3}\right) \in \boldsymbol{X}, \text { such that }\left(\boldsymbol{E}, E_{3}\right) \neq 0, \\
& \sup _{(\boldsymbol{F}, q) \in \boldsymbol{X}}\left|\boldsymbol{a}_{\omega}\left(\left(\boldsymbol{E}, E_{3}\right),(\boldsymbol{F}, q)\right)\right|>0 .
\end{aligned}
$$

We notice that (4.32) implies that $\beta=0$ cannot be in the spectrum of $\boldsymbol{a}_{\omega}$.

Spectral properties of the problem. As shown in [4] and [5], 4.1), 4.29), (4.30) imply that there are unique bounded solution operators $\hat{\boldsymbol{T}}: \mathcal{H} \longrightarrow \boldsymbol{X}$ and $\hat{\boldsymbol{T}}^{*}: \mathcal{H} \longrightarrow \boldsymbol{X}$ satisfying

$$
\begin{gathered}
\boldsymbol{a}_{\omega}(\hat{\boldsymbol{T}}(\boldsymbol{J}, Q),(\boldsymbol{F}, q))=((\boldsymbol{J}, Q),(\boldsymbol{F}, q))_{\mathcal{H}}, \quad(\boldsymbol{J}, Q) \in \mathcal{H},(\boldsymbol{F}, q) \in \boldsymbol{X}, \\
\boldsymbol{a}_{\omega}\left((\boldsymbol{F}, q), \hat{\boldsymbol{T}}^{*}(\boldsymbol{J}, Q)\right)=((\boldsymbol{F}, q),(\boldsymbol{J}, Q))_{\mathcal{H}}, \quad(\boldsymbol{F}, q) \in \boldsymbol{X},(\boldsymbol{J}, Q) \in \mathcal{H} .
\end{gathered}
$$

$\hat{\boldsymbol{T}}^{*}$ is the $\mathcal{H}$-adjoint of operator $\hat{\boldsymbol{T}}$. Furthermore, (4.1), (4.29) imply that

$$
\|\hat{\boldsymbol{T}}(\boldsymbol{J}, Q)\|_{\boldsymbol{X}} \leq \frac{C}{\alpha}\|(\boldsymbol{J}, Q)\|_{\epsilon} \quad \forall(\boldsymbol{J}, Q) \in \mathcal{H} .
$$

Theorem 4. $\hat{\boldsymbol{T}}: \mathcal{H} \longrightarrow \mathcal{H}$ is a compact operator.

Proof. First, 4.35) confirms that $\hat{\boldsymbol{T}}: \mathcal{H} \longrightarrow \mathcal{H}$ is a bounded operator. By Rellich's lemma the space $V$ is compactly embedded in $L^{2}(\Omega)$. We note that 4.35) alone is not sufficient to finish the proof, because $\boldsymbol{X}$ is not compactly embedded in $\mathcal{H}$ since $\boldsymbol{W}=H_{0}(\operatorname{curl}, \Omega)$ is not compact in $\boldsymbol{L}^{2}(\Omega)$. To overcome this difficulty, we notice that, for a given $(\boldsymbol{J}, Q) \in \mathcal{H}$, the pair $\left(\boldsymbol{E}, E_{3}\right)=\hat{\boldsymbol{T}}(\boldsymbol{J}, Q)$ must satisfy (4.18) $)_{2}$ adjusted as

$$
(\epsilon \boldsymbol{E}, \nabla q)=-\left(\epsilon E_{3}, q\right)+\frac{1}{\omega^{2}}(\epsilon Q, q), \quad \forall q \in V .
$$

Since $E_{3} \in V$ and $Q \in L^{2}(\Omega)$, using Green's formula in (4.36) we may conclude that $\operatorname{div}(\epsilon \boldsymbol{E}) \in L^{2}(\Omega)$, and there is $C>0$ such that

$$
\|\nabla \circ(\epsilon \boldsymbol{E})\|_{2} \leq C\|(\boldsymbol{J}, Q)\|_{\mathcal{H}} .
$$

Therefore, $\boldsymbol{E} \in H_{0}(\operatorname{curl}, \Omega) \cap H\left(\operatorname{div}_{\epsilon}, \Omega\right)$, which is indeed compactly embedded in $\boldsymbol{L}^{2}(\Omega)$, see [24 34]. Thus, we can show that $\boldsymbol{T}$ maps an $\mathcal{H}$-bounded set into an $\mathcal{H}$-compact one. Therefore, $\hat{\boldsymbol{T}}: \mathcal{H} \longrightarrow \mathcal{H}$ is a compact operator.

$\hat{\boldsymbol{T}}^{*}$ is also a compact operator on $\mathcal{H}$ as the adjoint of a compact operator, see [1]. It follows from (4.33) that $\boldsymbol{T}=\hat{\boldsymbol{T}} \mathbb{B}$, with $\mathbb{B}$ defined in (4.3), solves

$$
\boldsymbol{a}_{\omega}\left(T\left(\boldsymbol{E}, E_{3}\right),(\boldsymbol{F}, q)\right)=\left(\mathbb{B}\left(\boldsymbol{E}, E_{3}\right),(\boldsymbol{F}, q)\right)_{\mathcal{H}} \quad \forall\left(\boldsymbol{E}, E_{3}\right) \in \mathcal{H}, \forall(\boldsymbol{F}, q) \in \boldsymbol{X} .
$$

Considered on $\mathcal{H}, \boldsymbol{T}$ is a compact operator as the product of a bounded operator with a compact one. Using (4.38), we can rewrite (4.4) as

$$
\boldsymbol{a}_{\omega}\left(\left(1-\beta^{2} \boldsymbol{T}\right)\left(\boldsymbol{E}, E_{3}\right),(\boldsymbol{F}, q)\right)=0 \quad \forall\left(\boldsymbol{E}, E_{3}\right) \in \mathcal{H}, \forall(\boldsymbol{F}, q) \in \boldsymbol{X} .
$$

It follows immediately from (4.32) and (4.39) that the pair $\left(\beta,\left(\boldsymbol{E}, E_{3}\right)\right) \in \mathbb{C} \times \boldsymbol{X}$ solves (4.4) if and only if it solves the eigenvalue problem with the compact operator $T$

$$
\beta^{2} \boldsymbol{T}\left(\boldsymbol{E}, E_{3}\right)=\left(\boldsymbol{E}, E_{3}\right) .
$$


Thus, (4.40) means that $\lambda \neq 0$ is an eigenvalue of $\boldsymbol{T}$ if and only if $\lambda^{-1}$ is an eigenvalue of $\boldsymbol{a}_{\omega}$. So, by studying the spectral properties of (4.40) we can learn all we need to know about the spectral properties of $\boldsymbol{a}_{\omega}$.

As in [6], we introduce one more bounded operator ${ }^{2}$

$$
\boldsymbol{T}_{*}=\hat{\boldsymbol{T}}^{*} \mathbb{B} .
$$

$\boldsymbol{T}_{*}$ is compact on $\mathcal{H}$ as the composition of a bounded operator with a compact one. Since $\mathbb{B}$ is symmetric, we have

$$
\boldsymbol{a}_{\omega}\left((\boldsymbol{F}, q), \boldsymbol{T}_{*}\left(\boldsymbol{E}, E_{3}\right)=\left(\mathbb{B}(\boldsymbol{F}, q),\left(\boldsymbol{E}, E_{3}\right)\right)_{\mathcal{H}}, \quad \forall(\boldsymbol{F}, q) \in \boldsymbol{X}, \quad \forall\left(\boldsymbol{E}, E_{3}\right) \in \mathcal{H} .\right.
$$

Pairs $\left(\beta,\left(\boldsymbol{E}, E_{3}\right)\right) \in \mathbb{C} \times \boldsymbol{X}$ for the eigenvalue problem with $T_{*}$

$$
\bar{\beta}^{2} \boldsymbol{T}_{*}\left(\boldsymbol{E}, E_{3}\right)=\left(\boldsymbol{E}, E_{3}\right)
$$

correspond to the "so-called" adjoint eigenpairs of (4.4), see [6],

$$
\boldsymbol{a}_{\omega}\left((\boldsymbol{F}, q),\left(\boldsymbol{E}, E_{3}\right)\right)=\beta^{2}\left(\mathbb{B}(\boldsymbol{F}, q),\left(\boldsymbol{E}, E_{3}\right)\right)_{\mathcal{H}}, \quad \forall(\boldsymbol{F}, q) \in \boldsymbol{X} .
$$

The introduction of the adjoint eigenproblem (4.44) facilitates the convergence analysis for eigenvalues of the original problem (4.4).

For a short survey of spectral theory for compact operators, see [6] and the references therein. Since the eigenvalue problems with symmetric and nonsymmetric compact operators are rather different, we have decided to review the more important notions for our particular case. We denote by $\rho\left(\boldsymbol{T}_{\omega}\right)$ the resolvent set of $\boldsymbol{T}_{\omega}$, defined as

$$
\rho\left(\boldsymbol{T}_{\omega}\right)=\left\{z: z \in \mathbb{C}, \quad\left(z-\boldsymbol{T}_{\omega}\right)^{-1} \text { exists and it is bounded in } \mathcal{H}\right\}
$$

and by $\sigma\left(\boldsymbol{T}_{\omega}\right)$ the spectrum of $\boldsymbol{T}_{\omega}$, the set defined as

$$
\sigma\left(\boldsymbol{T}_{\omega}\right)=\mathbb{C} \backslash \rho\left(\boldsymbol{T}_{\omega}\right) .
$$

$\sigma\left(\boldsymbol{T}_{\omega}\right)$ is countable with no nonzero limit points. Since the space $\mathcal{H}$ is infinite dimensional, zero is in $\sigma\left(\boldsymbol{T}_{\omega}\right)$ as a limiting point. Nonzero elements of $\sigma\left(\boldsymbol{T}_{\omega}\right)$ are eigenvalues.

Let $\lambda \in \sigma\left(\boldsymbol{T}_{\omega}\right)$ be nonzero. We define the ascent of $\left(\lambda-\boldsymbol{T}_{\omega}\right)$ as the smallest integer $\kappa$ which satisfies

$$
\operatorname{Ker}\left(\left(\lambda-\boldsymbol{T}_{\omega}\right)^{\kappa}\right)=\operatorname{Ker}\left(\left(\lambda-\boldsymbol{T}_{\omega}\right)^{\kappa+1}\right),
$$

where Ker denotes the null space. The dimension of $\operatorname{Ker}\left(\left(\lambda-\boldsymbol{T}_{\omega}\right)^{\kappa}\right)$ is finite and the algebraic multiplicity of $\lambda$ is defined as

$$
m(\lambda)=\operatorname{dim}\left(\operatorname{Ker}\left(\left(\lambda-\boldsymbol{T}_{\omega}\right)^{\kappa}\right)\right) .
$$

The vectors in $\operatorname{Ker}\left(\left(\lambda-\boldsymbol{T}_{\omega}\right)^{\kappa}\right)$ are called generalized eigenvectors of $\boldsymbol{T}_{\omega}$ corresponding to $\lambda$.

The vectors in $\operatorname{Ker}\left(\lambda-\boldsymbol{T}_{\omega}\right)$ are called eigenvectors of $\boldsymbol{T}_{\omega}$ corresponding to $\lambda$.

The geometric multiplicity of $\lambda$ is defined as

$$
g(\lambda)=\operatorname{dim}\left(\operatorname{Ker}\left(\lambda-T_{\omega}\right)\right) .
$$

It is clear that $g(\lambda) \leq m(\lambda)$.

\footnotetext{
${ }^{2}$ If the operators $\hat{\boldsymbol{T}}^{*}$ and $\mathbb{B}$ commute, then $\boldsymbol{T}_{*}$ is the $\mathcal{H}$-adjoint of $\boldsymbol{T}$. As shown in the Appendix, in general, this is not the case.
} 
The sesquilinear form $\boldsymbol{a}_{\omega}$ is nonsymmetric, so the operator $\boldsymbol{T}_{\omega}$ is not self-adjoint and the elements of $\sigma\left(\boldsymbol{T}_{\omega}\right)$ are not necessarily real, and neither eigenvectors nor generalized eigenvectors are expected to form an orthogonal basis in $\mathcal{H}$.

\section{AnAlysis OF THE DISCRETIZED PROBLEM}

In this section we take $\Omega$ as a bounded polyhedral domain and consider a family of discretizations $\left\{\mathcal{T}_{h}\right\}_{h>0}$ by triangles or quadrilaterals. The discretization parameter $h$ usually denotes the maximum diameter of finite elements $K \in \mathcal{T}_{h}$.

We obtain a discretized version of eigenvalue problem (4.4) by taking test and trial functions not from $\boldsymbol{X}$ but from its subspace $\boldsymbol{X}_{h}=\boldsymbol{W}_{h} \times V_{h}$. We assume that the subspaces $\boldsymbol{W}_{h}$ and $V_{h}$ satisfy the following requirements:

- (R1): $\boldsymbol{W}_{h}$ and $V_{h}$ are compatible, in the sense that

$$
\boldsymbol{\nabla} V_{h}=\left\{\boldsymbol{E}_{h} \in \boldsymbol{W}_{h}: \boldsymbol{\nabla} \times \boldsymbol{E}_{h}=0\right\} .
$$

- $(\boldsymbol{R} 2)$ : $\boldsymbol{W}_{h}$ is discretely compact. This means, see [18], that any $\boldsymbol{W}$ bounded sequence $\left\{\boldsymbol{E}_{h}\right\}_{h>0}$ such that $\boldsymbol{E}_{h} \in \boldsymbol{W}_{h}$ and satisfying $\left(\epsilon \boldsymbol{E}_{h}, \boldsymbol{\nabla} q_{h}\right)$ $=0, \forall q_{h} \in V_{h}, \forall h$, contains a subsequence which converges weakly in $\boldsymbol{W}$ and strongly in $\boldsymbol{L}^{2}(\Omega)$ to an element $\boldsymbol{E} \in \boldsymbol{W}$.

- (R3): $\boldsymbol{W}_{h} \times V_{h}$ approximizes $\boldsymbol{W} \times V$ well:

$$
\lim _{h \longrightarrow 0} \inf _{\left(\boldsymbol{F}_{h}, q_{h}\right) \in \boldsymbol{X}_{h}}\left\|(\boldsymbol{E}, p)-\left(\boldsymbol{F}_{h}, q_{h}\right)\right\|_{\boldsymbol{X}}=0, \quad \forall(\boldsymbol{E}, p) \in \boldsymbol{X} .
$$

Notice that, in order to satisfy requirement $(\boldsymbol{R} 1)$, vector functions should be mapped from the master element into the mesh as gradients, see [16] 12] 33] for details.

Two families of edge elements have been shown to meet $(\boldsymbol{R} 2)$ : the widely used edge elements of Nedelec defined in [26, 27] and the recently introduced $h p$-adaptive generalization of Nedelec elements constructed in [14] and improved in [33], see also [12, 30. These elements allow local refinement in $h$ and enrichment in $p$. About a decade ago, Kikuchi proved the discrete compactness property for the Nedelec edge elements of lowest order defined on simplexes, see [19]. In [17], Joly extended that result to the case of nonconstant $\epsilon$ and $\mu$. Recently, Kikuchi has modified his proof to include the lowest order elements defined on quadrilaterals as well, see 20, 21. In 25, Monk et al., in the context of Maxwell's equations in $\mathbb{R}^{3}$ proved that the discrete compactness holds for Nedelec elements of any fixed order $p$. Some restrictive assumptions on the mesh were necessary in order for an inverse inequality to be applied. The results of that work remain valid for problems in two dimensions as well. In [13], Demkowicz et al. relaxed restrictions on the mesh. Moreover, the results were generalized to include the new family of $h p$-adaptive edge elements. The proof uses discrete compactness of the Nedelec elements of lowest order.

Remark 2. In [8], Boffi has linked $(\boldsymbol{R} 1)$ and $(\boldsymbol{R} 2)$ to the commuting property of the "de Rham complex" diagram which involves the spaces $H_{0}^{1}, \boldsymbol{H}_{0}(\mathrm{curl}), H_{0}$ (div), $L^{2} \backslash \mathbb{R}$ and their corresponding discretizations. Boffi has shown that this commuting property, combined with the uniform convergence of a particular projection operator, is in fact equivalent to the discrete compactness property. That the de Rham complex commutes for the standard edge elements has been known for a while, see [26, 3]. In [12], Demkowicz et al. have confirmed that it commutes for the $h p$-adaptive elements as well. 
In conjunction with the appropriately chosen conforming nodal approximation of $H^{1}(\Omega)$, both families satisfy $(\boldsymbol{R} 1)$ and $(\boldsymbol{R} 3)$ and can be employed in our analysis. Thus, the discretized version of eigenvalue problem (4.4) reads as follows.

For a given $\omega>0$ find $\left(\beta_{h},\left(\boldsymbol{E}_{h}, E_{3 h}\right)\right) \in\left(\mathbb{C}, \boldsymbol{X}_{h}\right)$ such that $\forall\left(\boldsymbol{F}_{h}, q_{h}\right) \in \boldsymbol{X}_{h}$

$$
\boldsymbol{a}_{\omega}\left(\left(\boldsymbol{E}_{h}, E_{3 h}\right),\left(\boldsymbol{F}_{h}, q_{h}\right)\right)=\beta_{h}^{2}\left(\mathbb{B}\left(\boldsymbol{E}_{h}, E_{3 h}\right),\left(\boldsymbol{F}_{h}, q_{h}\right)\right)_{\mathcal{H}} .
$$

We note that, for a fixed $h>0$, utilizing $(\boldsymbol{R} 1)$, we can repeat the steps of the argument leading to the proof of the continuous inf - sup conditions (4.29), (4.30) and obtain the discretized inf - sup constant

$$
\alpha_{h}=C \gamma_{1 h} \gamma_{2 h},
$$

where $C>0$ is some constant and

$$
\gamma_{1 h}=\min _{i=1 \ldots \infty}\left\{\frac{\left|\eta_{i h}-\omega^{2}\right|}{\eta_{i h}}\right\}
$$

with $\eta_{i h}$ being the eigenvalues of the discretized eigenproblem on $\nabla \frac{1}{\mu} \nabla$ (3.14) considered on $V_{h}$, and

$$
\gamma_{2 h}=\min \left\{\min _{j=1 \ldots \infty}\left\{\frac{\left|\nu_{j h}-\omega^{2}\right|}{\nu_{j h}}\right\}, \frac{1}{1+\omega^{2}}\right\}
$$

with $\nu_{j h}$ being eigenvalues of the discretized eigenproblem on $\nabla \times \frac{1}{\mu} \nabla \times$ considered on $\boldsymbol{W}_{h}$.

By the classical theory of $H^{1}$-conforming discretizations of the Laplace operator we get

$$
\lim _{h \rightarrow 0} \eta_{i h}=\eta_{i}, \quad \forall i \geq 1
$$

and, using the recent results in [25, 13, 9, 15, applicable to our problem, owing to $(\boldsymbol{R} 2)$, we may conclude that

$$
\lim _{h \rightarrow 0} \nu_{j h}=\nu_{j}, \quad \forall j \geq 1 .
$$

Since all $\eta_{i}$ and $\nu_{j}$ are positive, $\eta_{i h}^{-1}$ and $\nu_{j h}^{-1}$ remain bounded as $h \rightarrow 0$. Therefore, as a direct consequence of Theorem 3 and (5.4)-(5.8) we have

Theorem 5. With the assumption on $\omega$ as in 3.15, there is $h_{0}>0$ such that $\forall h>h_{0}$ the form $\boldsymbol{a}_{\omega}$ satisfies the following conditions:

$$
\begin{aligned}
& \exists \alpha_{0}>0, \text { such that } \forall \alpha_{h} \geq \alpha_{0} \\
& \sup _{\left(\boldsymbol{F}_{h}, q_{h}\right) \in \boldsymbol{X}_{h}} \frac{\left|\boldsymbol{a}_{\omega}\left(\left(\boldsymbol{E}_{h}, E_{3 h}\right),\left(\boldsymbol{F}_{h}, q_{h}\right)\right)\right|}{\left\|\left(\boldsymbol{F}_{h}, q_{h}\right)\right\|_{\boldsymbol{X}}} \\
& \quad \geq \alpha_{0}\left\|\left(\boldsymbol{E}_{h}, E_{3 h}\right)\right\|_{\boldsymbol{X}} \quad \forall\left(\boldsymbol{E}_{h}, E_{3 h}\right) \in \boldsymbol{X}_{h}
\end{aligned}
$$

and

$$
\begin{aligned}
& \forall\left(\boldsymbol{F}_{h}, q_{h}\right) \in \boldsymbol{X}_{h}, \text { such that }\left(\boldsymbol{F}_{h}, q_{h}\right) \neq 0 \\
& \sup _{\left(\boldsymbol{E}_{h}, E_{3 h}\right) \in \boldsymbol{X}_{h}}\left|\boldsymbol{a}_{\omega}\left(\left(\boldsymbol{E}_{h}, E_{3 h}\right),\left(\boldsymbol{F}_{h}, q_{h}\right)\right)\right|>0 .
\end{aligned}
$$


Inequality (5.9) is called the discrete inf - sup condition. We also automatically get

$$
\sup _{\left(\boldsymbol{E}_{h}, E_{3 h}\right) \in \boldsymbol{X}_{h}} \frac{\left|\boldsymbol{a}_{\omega}\left(\left(\boldsymbol{E}_{h}, E_{3 h}\right),\left(\boldsymbol{F}_{h}, q_{h}\right)\right)\right|}{\left\|\left(\boldsymbol{F}_{h}, q_{h}\right)\right\|_{\boldsymbol{X}}} \geq \alpha_{0}\left\|\left(\boldsymbol{E}_{h}, E_{3 h}\right)\right\|_{\boldsymbol{X}} \quad \forall\left(\boldsymbol{F}_{h}, q_{h}\right) \in \boldsymbol{X}_{h}
$$

and

$$
\begin{aligned}
& \forall\left(\boldsymbol{E}_{h}, E_{3 h}\right) \in \boldsymbol{X}_{h}, \text { such that }\left(\boldsymbol{E}_{h}, E_{3 h}\right) \neq 0, \\
& \sup _{\left(\boldsymbol{F}_{h}, q_{h}\right) \in \boldsymbol{X}_{h}}\left|\boldsymbol{a}_{\omega}\left(\left(\boldsymbol{E}_{h}, E_{3 h}\right),\left(\boldsymbol{F}_{h}, q_{h}\right)\right)\right|>0 .
\end{aligned}
$$

We notice that (5.12) implies that $\beta_{h}=0$ is not in the spectrum of (5.3). Just as for the continuous problem, (5.9) and (5.10) allow us to introduce a pair of compact operators, $\hat{\boldsymbol{T}}_{h}$ and its $\mathcal{H}$-adjoint $\hat{\boldsymbol{T}}_{h}{ }^{*}$, defined uniquely by

$$
\boldsymbol{a}_{\omega}\left(\hat{\boldsymbol{T}}_{h}(\boldsymbol{J}, Q),\left(\boldsymbol{F}_{h}, q_{h}\right)\right)=\left((\boldsymbol{J}, Q),\left(\boldsymbol{F}_{h}, q_{h}\right)\right)_{\mathcal{H}}, \quad \forall(\boldsymbol{J}, Q) \in \mathcal{H}, \quad \forall\left(\boldsymbol{F}_{h}, q_{h}\right) \in \boldsymbol{X}_{h}
$$

and

$\boldsymbol{a}_{\omega}\left(\left(\boldsymbol{F}_{h}, q_{h}\right), \hat{\boldsymbol{T}}_{h}{ }^{*}(\boldsymbol{J}, Q)\right)=\left(\left(\boldsymbol{F}_{h}, q_{h}\right),(\boldsymbol{J}, Q)\right)_{\mathcal{H}}, \quad \forall\left(\boldsymbol{F}_{h}, q_{h}\right) \in \boldsymbol{X}_{h}, \quad \forall(\boldsymbol{J}, Q) \in \mathcal{H}$.

The operators $\hat{\boldsymbol{T}}_{h}$ and $\hat{\boldsymbol{T}}^{*}{ }_{h}$ are compact since they both have finite range. And, as on the continuous level, using (5.13) and (5.12) we can show that (5.3) is equivalent to

$$
\left(\boldsymbol{E}_{h}, E_{3 h}\right)=\beta_{h}^{2} \boldsymbol{T}_{h}\left(\boldsymbol{E}_{h}, E_{3 h}\right),
$$

where $\boldsymbol{T}_{h}: \mathcal{H} \longrightarrow \mathcal{H}$ is defined by

$$
\boldsymbol{T}_{h}=\hat{\boldsymbol{T}}_{h} \mathbb{B}
$$

and $\mathbb{B}$ comes from (4.3). Since any finite element method produces eigenvalues and eigenvectors which correspond to $\boldsymbol{T}_{h}$, we need to analyze how well the spectral characteristics of $\boldsymbol{T}_{h}$ reflect the spectral characteristics of $\boldsymbol{T}$, as $h \rightarrow 0$. We address this issue in the next section.

\section{Convergence analysis}

The convergence analysis, performed in the framework of collectively compact operators as in [17] 25], relies on the following theorem (slightly adjusted to fit our case) by Chatelin, see [10].

Theorem 6. Let us consider a family of operators $\left\{\boldsymbol{T}_{h}\right\}_{0<h<h_{0}}$ with the corresponding $\mathcal{H}$-adjoints $\left\{\boldsymbol{T}_{h}^{*}\right\}_{0<h<h_{0}}$ converging pointwise to compact operators $\boldsymbol{T}$ and $\boldsymbol{T}^{*}$, resp., i.e.,

$$
\begin{array}{ll}
\lim _{h \rightarrow 0}\left\|\boldsymbol{T}_{h}(\boldsymbol{J}, Q)-\boldsymbol{T}(\boldsymbol{J}, Q)\right\|_{\mathcal{H}}=0 & \forall(\boldsymbol{J}, Q) \in \mathcal{H}, \\
\lim _{h \rightarrow 0}\left\|\boldsymbol{T}_{h}^{*}(\boldsymbol{J}, Q)-\boldsymbol{T}^{*}(\boldsymbol{J}, Q)\right\|_{\mathcal{H}}=0 & \forall(\boldsymbol{J}, Q) \in \mathcal{H} .
\end{array}
$$

Moreover, assume that the family $\left\{\boldsymbol{T}_{h}\right\}_{0<h<h_{0}}$ is collectively compact, i.e., the set

$$
K=\left\{\boldsymbol{T}_{h}(\boldsymbol{J}, Q):\|(\boldsymbol{J}, Q)\|_{\mathcal{H}} \leq 1\right\}_{0<h<h_{0}}
$$


is sequentially compact in $\mathcal{H}$. Then $\boldsymbol{T}_{h} \rightarrow \boldsymbol{T}$ in the norm

$$
\lim _{h \rightarrow 0}\left\|\boldsymbol{T}_{h}-\boldsymbol{T}\right\|_{\mathcal{L}(\mathcal{H}, \mathcal{H})}=0 .
$$

Proof. Let us suppose that assertion (6.4) is false; then there exist a sequence $\left\{\left(\boldsymbol{J}_{h}, Q_{h}\right):\left\|\left(\boldsymbol{J}_{h}, Q_{h}\right)\right\|_{\mathcal{H}} \leq 1\right\}_{0<h<h_{0}}$ and $\delta$ such that

$$
\left\|\left(\boldsymbol{T}-\boldsymbol{T}_{h}\right)\left(\boldsymbol{J}_{h}, Q_{h}\right)\right\|_{\mathcal{H}} \geq \delta,
$$

and, since the set $K$ is sequentially compact and $\boldsymbol{T}$ is a compact operator, there exists $(F, q) \in \mathcal{H}$ such that

$$
\lim _{h \rightarrow 0}\left(\boldsymbol{T}-\boldsymbol{T}_{h}\right)\left(\boldsymbol{J}_{h}, Q_{h}\right)=(\boldsymbol{F}, q), \quad\|(\boldsymbol{F}, q)\|_{\mathcal{H}} \geq \delta .
$$

Now let $\left(\boldsymbol{F}^{*}, q^{*}\right) \in \mathcal{H}$ be scaled to yield

$$
\|(\boldsymbol{F}, q)\|_{\mathcal{H}}=\left(\left(\boldsymbol{F}^{*}, q^{*}\right),(\boldsymbol{F}, q)\right)_{\mathcal{H}} .
$$

Then

$$
\begin{aligned}
\|(\boldsymbol{F}, q)\|_{\mathcal{H}} & =\lim _{h \rightarrow 0}\left(\left(\boldsymbol{F}^{*}, q^{*}\right),\left(\boldsymbol{T}-\boldsymbol{T}_{h}\right)\left(\boldsymbol{J}_{h}, Q_{h}\right)\right)_{\mathcal{H}} \\
& =\lim _{h \rightarrow 0}\left(\left(\boldsymbol{T}^{*}-\boldsymbol{T}_{h}^{*}\right)\left(\boldsymbol{F}^{*}, q^{*}\right),\left(\boldsymbol{J}_{h}, Q_{h}\right)\right)_{\mathcal{H}}=0,
\end{aligned}
$$

since we assumed pointwise convergence of $\boldsymbol{T}_{h}^{*}$. Clearly, (6.8) contradicts $\|(\boldsymbol{F}, q)\|_{\mathcal{H}}$ $\geq \delta$. Therefore, 6.4 must be true.

Thus, if we confirm properties (6.1)- 6.3 , all of Osborn's theorems on convergence in 29] become applicable.

6.1. Pointwise convergence of $\left\{\boldsymbol{T}_{h}\right\}_{0<h<h_{0}}$ and $\left\{\boldsymbol{T}_{h}^{*}\right\}_{0<h<h_{0}}$. We note that since $\mathbb{B}$ is bounded it is sufficient to verify pointwise convergence of $\left\{\hat{\boldsymbol{T}}_{h}\right\}_{0<h<h_{0}}$ and $\left\{\hat{\boldsymbol{T}}_{h}^{*}\right\}_{0<h<h_{0}}$.

Using the standard convergence theory of the finite element method and (5.9), we can show that, see [5],

$$
\left\|\hat{\boldsymbol{T}}(\boldsymbol{J}, Q)-\hat{\boldsymbol{T}}_{h}(\boldsymbol{J}, Q)\right\|_{\boldsymbol{X}} \leq\left(1+\frac{C}{\alpha_{0}}\right) \inf _{\left(\boldsymbol{F}_{h}, q_{h}\right) \in \boldsymbol{X}_{h}}\left\|\hat{\boldsymbol{T}}(\boldsymbol{J}, Q)-\left(\boldsymbol{F}_{h}, q_{h}\right)\right\|_{\boldsymbol{X}}
$$

Now we recall that $\boldsymbol{X}_{h}$ satisfies "approximability requirement" ( $\left.R 3\right)$. Therefore it follows from 6.9 that

$$
\lim _{h \rightarrow 0}\left\|\hat{\boldsymbol{T}}(\boldsymbol{J}, Q)-\hat{\boldsymbol{T}}_{h}(\boldsymbol{J}, Q)\right\|_{\boldsymbol{X}}=0, \quad \forall(\boldsymbol{J}, Q) \in \mathcal{H} .
$$

And since the $\boldsymbol{X}$-norm is stronger that the $\mathcal{H}$-norm, we confirm pointwise convergence of $\hat{\boldsymbol{T}}_{h}$ in $\mathcal{H}$ :

$$
\lim _{h \rightarrow 0}\left\|\hat{\boldsymbol{T}}(\boldsymbol{J}, Q)-\hat{\boldsymbol{T}}_{h}(\boldsymbol{J}, Q)\right\|_{\mathcal{H}}=0, \quad \forall(\boldsymbol{J}, Q) \in \mathcal{H}
$$

Applying the same argument and (5.11), we verify pointwise convergence of $\hat{\boldsymbol{T}}_{h}^{*}$ in $\mathcal{H}$ :

$$
\lim _{h \rightarrow 0}\left\|\hat{\boldsymbol{T}}^{*}(\boldsymbol{J}, Q)-\hat{\boldsymbol{T}}_{h}^{*}(\boldsymbol{J}, Q)\right\|_{\mathcal{H}}=0, \quad \forall(\boldsymbol{J}, Q) \in \mathcal{H}
$$


6.2. Collective compactness of $\left\{\boldsymbol{T}_{h}\right\}_{0<h<h_{0}}$. Let us consider a closed unit ball in $\mathcal{H}$,

$$
S=\left\{(\boldsymbol{J}, Q) \in \mathcal{H}:\|(\boldsymbol{J}, Q)\|_{\mathcal{H}} \leq 1\right\} .
$$

The set $K$ defined in (6.3) can be described as

$$
K=\bigcup_{0<h<h_{0}}\left\{\boldsymbol{T}_{h}(\boldsymbol{J}, Q):(\boldsymbol{J}, Q) \in S\right\} .
$$

We show that any sequence $\left\{\left(\boldsymbol{E}_{h}, E_{3 h}\right)\right\}_{0<h<h_{0}} \in K$ contains a subsequence converging strongly in $\mathcal{H}$ in three steps.

Step 1. From (6.10), the Banach-Steinhaus Theorem, and the boundedness of $\mathbb{B}$, we may conclude that

$$
\left\|\boldsymbol{T}_{h}\right\|_{\mathcal{L}(\mathcal{H}, \boldsymbol{X})} \leq C, \quad 0<h<h_{0},
$$

where $C$ is some positive constant. Therefore,

$$
\left\|\left(\boldsymbol{E}_{h}, E_{3 h}\right)\right\|_{\boldsymbol{X}} \leq C, \quad \forall\left(\boldsymbol{E}_{h}, E_{3 h}\right) \in K .
$$

This means that

$$
\left\|\boldsymbol{E}_{h}\right\|_{\boldsymbol{W}} \leq C \quad \text { and } \quad\left\|E_{3 h}\right\|_{V} \leq C .
$$

We recall now that $V$ is compactly embedded in $L_{\epsilon}^{2}(\Omega)$. Consequently, any sequence $\left\{\left(\boldsymbol{E}_{h}, E_{3 h}\right)\right\} \in K$ contains a subsequence (with elements still marked by $h$ ) such that

$$
\lim _{h \rightarrow 0}\left\|E_{3 h}-E_{3}\right\|_{\epsilon}=0
$$

where $E_{3}$ is some element of $V$. Let us consider this subsequence in further detail. Since $\left(\boldsymbol{E}_{h}, E_{3 h}\right)$ are in $K$, then

$$
\left(\boldsymbol{E}_{h}, E_{3 h}\right)=\boldsymbol{T}_{h}(\boldsymbol{J}, Q)
$$

for some $(\boldsymbol{J}, Q) \in \mathcal{H}$. It follows from (4.3) and (4.4) that all $\left(\boldsymbol{E}_{h}, E_{3 h}\right)$ must satisfy

$$
\left\{\begin{aligned}
&\left(\frac{1}{\mu} \boldsymbol{\nabla} \times \boldsymbol{E}_{h}, \boldsymbol{\nabla} \times \boldsymbol{F}_{h}\right)-\omega^{2}\left(\epsilon \boldsymbol{E}_{h}, \boldsymbol{F}_{h}\right) \\
&=\left(\frac{1}{\mu} \boldsymbol{\nabla} E_{3 h}, \boldsymbol{F}_{h}\right)-\left(\frac{1}{\mu} \boldsymbol{J}, \boldsymbol{F}_{h}\right) \quad \forall \boldsymbol{F}_{h} \in \boldsymbol{W}_{h} \\
&\left(\epsilon \boldsymbol{E}_{h}, \boldsymbol{\nabla} q_{h}\right)=-\left(\epsilon E_{3 h}, q_{h}\right) \quad \forall q_{h} \in V_{h}
\end{aligned}\right.
$$

where $E_{3 h}$ is known as the solution to

$$
-\left(\frac{1}{\mu} \nabla E_{3 h}, \nabla q_{h}\right)+\omega^{2}\left(\epsilon E_{3 h}, q_{h}\right)=-\left(\frac{1}{\mu} \boldsymbol{J}, \nabla q_{h}\right) \quad \forall q_{h} \in V_{h} .
$$

Step 2. To facilitate further analysis, we would like to split $\boldsymbol{E}_{h}$ into the curl-free part and the discrete divergence-free part. We can accomplish this task by using $(6.20) 2$.

First, we note that the function $g_{h} \in V_{h}$ is uniquely defined as the solution to

$$
\left(\epsilon \boldsymbol{\nabla} g_{h}, \boldsymbol{\nabla} q_{h}\right)=-\left(\epsilon E_{3 h}, q_{h}\right), \quad \forall q_{h} \in V_{h},
$$

and $E_{3 h}$ is already known.

Now, if we introduce $\boldsymbol{w}_{h} \in \boldsymbol{W}_{h}$ as

$$
\boldsymbol{w}_{h}=\boldsymbol{E}_{h}-\nabla g_{h},
$$


then it follows from $(6.20) 2$ and $(6.22)$ that $\boldsymbol{w}_{h}$ is indeed discrete divergence free:

$$
\left(\epsilon \boldsymbol{w}_{h}, \boldsymbol{\nabla} q_{h}\right)=0, \quad \forall q_{h} \in V_{h} .
$$

Thus, by (6.23) we have the needed decomposition

$$
\boldsymbol{E}_{h}=\boldsymbol{\nabla} g_{h}+\boldsymbol{w}_{h}
$$

We also consider an auxiliary problem on $\hat{g}_{h}$ derived from (6.22) with $E_{3 h}$ on the right-hand side replaced by $E_{3}$ :

$$
\left(\epsilon \boldsymbol{\nabla} \hat{g}_{h}, \boldsymbol{\nabla} q_{h}\right)=-\left(\epsilon E_{3}, q_{h}\right), \quad \forall q_{h} \in V_{h} .
$$

By subtracting (6.26) from (6.22), taking $q_{h}=g_{h}-\hat{g}_{h}$ and applying the Poincaré inequality, we can show that there is a $C>0$ such that

$$
\left\|g_{h}-\hat{g}_{h}\right\|_{V} \leq C\left\|E_{3}-E_{3 h}\right\|_{\epsilon} \quad \forall h>0 .
$$

Using the standard theory of $H^{1}$-conforming approximation for elliptic equations, we can deduce that there exists a $g \in V$ such that $\hat{g}_{h}$, the solution to (6.26), satisfies

$$
\lim _{h \rightarrow 0}\left\|g-\hat{g}_{h}\right\|_{V}=0 .
$$

From (6.27), combined with (6.18) and (6.28), using the triangle inequality, we readily get

$$
\lim _{h \rightarrow 0}\left\|\nabla g-\nabla g_{h}\right\|_{\epsilon}=0 .
$$

And, since a converging sequence is always bounded,

$$
\left\|\nabla g_{h}\right\|_{\epsilon} \leq C, \quad 0<h<h_{0},
$$

where $C$ is some positive constant, we have shown that the curl-free part of $\boldsymbol{E}_{h}$ converges in $\boldsymbol{L}_{\epsilon}^{2}(\Omega)$. The discrete compactness property $(\boldsymbol{R} 2)$ is necessary to make the remaining part of $\boldsymbol{E}_{h}$ converge as well.

Step 3. By its definition, $\boldsymbol{w}_{h}$ is expressed as $\boldsymbol{w}_{h}=\boldsymbol{E}_{h}-\boldsymbol{\nabla} g_{h}$. Moreover, we have established separate bounds on $\boldsymbol{E}_{h}$ and $\boldsymbol{\nabla} g_{h}$ in (6.17) and (6.30). Therefore, by the triangle inequality,

$$
\left\|\boldsymbol{w}_{h}\right\|_{\epsilon} \leq\left\|\boldsymbol{w}_{h}\right\|_{\boldsymbol{W}}<C, \quad 0<h<h_{0},
$$

where $C$ is some positive constant. Now, since the spaces $\boldsymbol{W}_{h}$ satisfy $(\boldsymbol{R} 2)$, there exists a subsequence of $\left\{\left(\boldsymbol{E}_{h}, E_{3 h}\right)\right\}$ (still marked by $h$ ) whose elements possess discrete divergence free parts $\boldsymbol{w}_{h}$ converging strongly in $\boldsymbol{L}_{\epsilon}^{2}(\Omega)$ :

$$
\lim _{h \rightarrow 0}\left\|\boldsymbol{w}_{h}-\boldsymbol{w}\right\|_{\epsilon}=0,
$$

where $\boldsymbol{w}$ is some element in $\boldsymbol{W}$. It follows from (6.18), (6.29), (6.32), and decomposition (6.25) that this subsequence converges strongly in $\mathcal{H}$ :

$$
\lim _{h \rightarrow 0}\left\|\left(\boldsymbol{E}_{h}, E_{3 h}\right)-\left(\boldsymbol{E}, E_{3}\right)\right\|_{\mathcal{H}}=0,
$$

where $\boldsymbol{E}=\boldsymbol{w}+\boldsymbol{\nabla} g$ of (6.32) and (6.28), and $E_{3}$ comes from (6.18). Thus, the set $K$ is sequentially compact, and collective compactness has been confirmed. Therefore, by theorem 6 we get convergence in the norm: $\lim _{h \rightarrow 0}\left\|\boldsymbol{T}-\boldsymbol{T}_{h}\right\|_{\mathcal{L}(\mathcal{H}, \mathcal{H})}=0$. 
6.3. Convergence estimates. Let $\lambda$ be a nonzero eigenvalue of (4.4) with algebraic multiplicity $m$. This also means that $\lambda^{-1}$ is an eigenvalue of $\boldsymbol{T}$, with equal multiplicity. And let us assume that the ascent of $\lambda^{-1}-\boldsymbol{T}$ is $\kappa$. We denote by $\boldsymbol{E}$ the spectral projection (see e.g., [6]) associated with $\lambda^{-1}$ and $\boldsymbol{T}$, and by $R(\boldsymbol{E})$ its range. $\boldsymbol{E}^{*}$ denotes the spectral projection associated with $\bar{\lambda}^{-1}$ and $\boldsymbol{T}^{*}, R\left(\boldsymbol{E}^{*}\right)$ being the range of $\boldsymbol{E}^{*}$.

Like Babuška and Osborn in [6], we introduce the following two sets related to the eigenproblems (4.4) and (4.44) on $\boldsymbol{a}_{\omega}$ :

$$
\begin{array}{r}
S=S(\lambda)=\left\{\left(\boldsymbol{E}, E_{3}\right):\left(\boldsymbol{E}, E_{3}\right)\right. \text { is a generalized eigenvector of (4.4) } \\
\text { corresponding to } \left.\lambda,\left\|\left(\boldsymbol{E}, E_{3}\right)\right\|_{\mathcal{H}}=1\right\},
\end{array}
$$

$$
\begin{gathered}
S^{*}=S^{*}(\lambda)=\left\{\left(\boldsymbol{E}^{*}, E_{3}^{*}\right):\left(\boldsymbol{E}^{*}, E_{3}^{*}\right)\right. \text { is a generalized adjoint eigenvector of (4.4) } \\
\text { corresponding to } \left.\lambda,\left\|\left(\boldsymbol{E}^{*}, E_{3}^{*}\right)\right\|_{\mathcal{H}}=1\right\},
\end{gathered}
$$

and since such $\left(\boldsymbol{E}, E_{3}\right)$ and $\left(\boldsymbol{E}^{*}, E_{3}^{*}\right)$ are in $\boldsymbol{X}$, let us also define

$$
\varepsilon_{h}=\varepsilon_{h}(\lambda)=\sup _{\left(\boldsymbol{E}, E_{3}\right) \in S(\lambda)} \inf _{\left(\boldsymbol{F}_{h}, q_{h}\right) \in \boldsymbol{X}_{h}}\left\|\left(\boldsymbol{E}, E_{3}\right)-\left(\boldsymbol{F}_{h}, q_{h}\right)\right\|_{\boldsymbol{X}}
$$

and

$$
\varepsilon_{h}^{*}=\varepsilon_{h}^{*}(\lambda)=\sup _{\left(\boldsymbol{E}^{*}, E_{3}^{*}\right) \in S^{*}(\lambda)} \inf _{\left(\boldsymbol{F}_{h}, q_{h}\right) \in \boldsymbol{X}_{h}}\left\|\left(\boldsymbol{E}^{*}, E_{3}^{*}\right)-\left(\boldsymbol{F}_{h}, q_{h}\right)\right\|_{\boldsymbol{X}} .
$$

In 29] Osborn proves

Theorem 7. Consider a disc centered at $\lambda^{-1}$, of radius $\epsilon>0$ such that it contains no other eigenvalue of $\boldsymbol{T}$. Then there is an $\hat{h}>0$ such that, for any $0<h<\hat{h}$, this disc contains exactly $m$ eigenvalues $\lambda_{i, h}^{-1}, i=1, \ldots, m$ (counted with multiplicity), of $\boldsymbol{T}_{h}$, and there is a constant $C>0$ such that for any $i=1, \ldots, m$

$$
\begin{aligned}
& \frac{\left|\lambda^{-1}-\lambda_{i, h}^{-1}\right|^{\kappa}}{C} \leq \sum_{j, l=1}^{m}\left|\left(\left(\boldsymbol{T}-\boldsymbol{T}_{h}\right) \phi_{j}, \phi_{l}^{\prime}\right){ }_{\mathcal{H}}\right| \\
& +\left\|\left.\left(\boldsymbol{T}-\boldsymbol{T}_{h}\right)\right|_{R(\boldsymbol{E})}\right\|_{\mathcal{L}(\mathcal{H}, \mathcal{H})}\left\|\left.\left(\boldsymbol{T}^{*}-\boldsymbol{T}_{h}^{*}\right)\right|_{R\left(\boldsymbol{E}^{*}\right)}\right\|_{\mathcal{L}(\mathcal{H}, \mathcal{H})} .
\end{aligned}
$$

Here $\left\{\phi_{j}\right\}_{j=1}^{m}$ is any basis for $R(\boldsymbol{E})$ and $\left\{\phi_{j}^{\prime}\right\}_{j=1}^{m}$ is the dual basis with respect to the $\mathcal{H}$-inner product.

It is clear that since $\lambda$ is nonzero, the estimate (6.38) also applies to $\left|\lambda-\lambda_{i, h}\right|^{\kappa}$ (with a different $C$ ).

Remark 3. Osborn has shown that vectors $\left\{\phi_{j}^{\prime}\right\}_{j=1}^{m}$ can be extended to all of $\mathcal{H}$ as the generalized eigenvectors of $\boldsymbol{T}^{*}$ corresponding to $\bar{\lambda}^{-1}$.

In [6], Babuška and Osborn use Theorem[7to derive convergence estimates which involve $\varepsilon_{h}$ and $\varepsilon_{h}^{*}$ defined in (6.36) and (6.37), which are evidently closely related to the original eigenvalue problem (4.4). Their approach has to be adjusted to the current case because we did not demonstrate compactness of $\boldsymbol{T}$ on the space $\boldsymbol{X}$, where the isomorphism $L$ of (4.5), corresponding to the form $\boldsymbol{a}_{\omega}$ is defined. We 
consider this matter in full detail in the Appendix. There we show that

$$
\begin{aligned}
& \sum_{j, l=1}^{m}\left|\left(\left(\boldsymbol{T}-\boldsymbol{T}_{h}\right) \phi_{j}, \phi_{l}^{\prime}\right)_{\mathcal{H}}\right| \\
& \quad+\left\|\left.\left(\boldsymbol{T}-\boldsymbol{T}_{h}\right)\right|_{R(\boldsymbol{E})}\right\|_{\mathcal{L}(\mathcal{H}, \mathcal{H})}\left\|\left.\left(\boldsymbol{T}^{*}-\boldsymbol{T}_{h}^{*}\right)\right|_{R\left(\boldsymbol{E}^{*}\right)}\right\|_{\mathcal{L}(\mathcal{H}, \mathcal{H})} \leq \frac{C}{\alpha_{0}} \varepsilon_{h} \varepsilon_{h}^{*},
\end{aligned}
$$

where $C$ is some positive constant. Thus, we arrive at our final convergence result for the eigenvalue problem on $\boldsymbol{a}_{\omega}$ :

$$
\left|\lambda-\lambda_{i, h}\right|^{\kappa}=\mathcal{O}\left(\varepsilon_{h} \varepsilon_{h}^{*}\right),
$$

which shows that the rate of convergence depends upon the ascent $\kappa$ and the interpolation error estimates for the eigenspaces.

6.4. Convergence rates for Nedelec elements. The estimates of convergence rates are necessary for the analysis and design of successful adaptive schemes. Since the $h p$-edge elements [14, 33] generalize Nedelec elements [26, 27, we consider the Nedelec elements first, with the intention of using these results for further study of the $h p$-convergence mechanism and $h p$-adaptivity. To get the rates of convergence for a propagation constant $\beta$, we need to know the regularity of the eigenmodes $\left(\boldsymbol{E}, E_{3}\right)$ and the adjoint eigenmodes $\left(\boldsymbol{E}^{*}, E_{3}^{*}\right)$ which correspond to $\beta^{2}$, see [6]. Let us assume that all the eigenmodes and the adjoint eigenmodes for $\beta^{2}$ of multiplicity $m$ and ascent $\kappa$ are such that

$$
\begin{array}{lll}
\boldsymbol{E} \in \boldsymbol{H}^{r}(\Omega), & \boldsymbol{\nabla} \times \boldsymbol{E} \in \boldsymbol{H}^{r}(\Omega), & E_{3} \in H^{r+1}(\Omega), \\
\boldsymbol{E}^{*} \in \boldsymbol{H}^{r}(\Omega), & \boldsymbol{\nabla} \times \boldsymbol{E}^{*} \in \boldsymbol{H}^{r}(\Omega), & E_{3}^{*} \in H^{r+1}(\Omega),
\end{array}
$$

where $\boldsymbol{H}^{r}$ is Sobolev space of order $r>0$. If $H^{1}$-conforming scalar elements of order $p$ are used in shape-regular affine meshes with $N=N(h)$ degrees of freedom, then, slightly adjusting the proofs given in $[2$ to the $2 \mathrm{D}$ case, we can bound the interpolation error and, consequently, estimate the eigenvalue rate of convergence as follows:

$$
\begin{aligned}
& \left\|(\boldsymbol{E}, E)-\left(\boldsymbol{E}_{h}, E_{3, h}\right)\right\|_{\boldsymbol{X}} \leq \mathcal{O}\left(N^{-\frac{s}{2}}\right), \\
& \left|\beta-\beta_{i, h}\right|^{\kappa} \leq \mathcal{O}\left(N^{-s}\right),
\end{aligned}
$$

where $s=\min (p, r)$, provided that the compatible Nedelec triangles or quads of the first family [26] are used. In general, if the compatible Nedelec triangles of the second family 27] are used, then $s=\min (p-1, r)$. However, if we solve for $\left(\boldsymbol{E}, E_{3}\right)$ and the modes corresponding to $\beta^{2}$ are all TM or TEM, or if we solve for $\left(\boldsymbol{H}, H_{3}\right)$ and all the modes are TE or TEM, then the triangles of both families offer the same rate of convergence.

\section{SAMPLE NUMERICAL EXAMPLES}

We confirm convergence rates (6.42) by applying our method to homogeneously loaded waveguides with known exact solutions derived from eigenfunctions for the Laplace operator with Dirichlet or Neumann boundary conditions. For all considered cases, solution operators are such that ascent $\kappa=1$, and all eigenmodes and the corresponding adjoint eigenmodes have the same regularity. To confirm (6.42), we implement Nedelec quads of [26] and Nedelec triangles of [27] on uniform meshes. We use 2Dhp90_EM, a FE package for electromagnetics [30], designed to support the $h p$-edge elements which generalize these quads and triangles of Nedelec. 


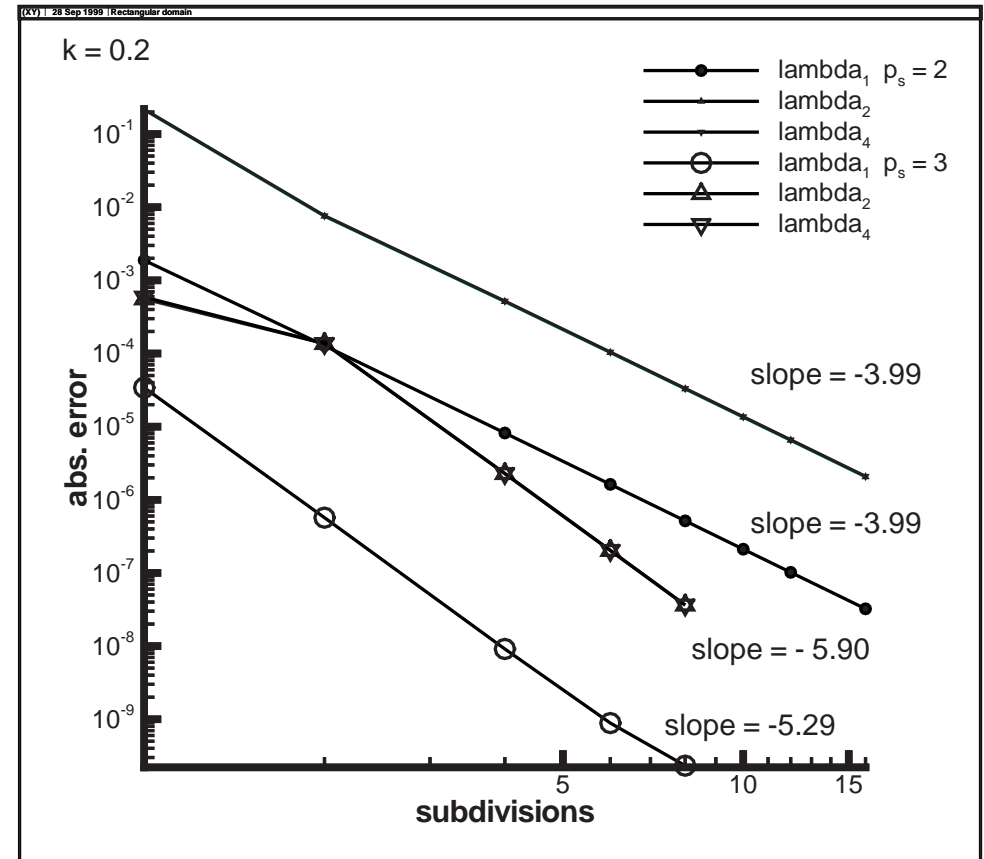

FiguRE 1. Dielectric rectangle: $h$-refinement by quads

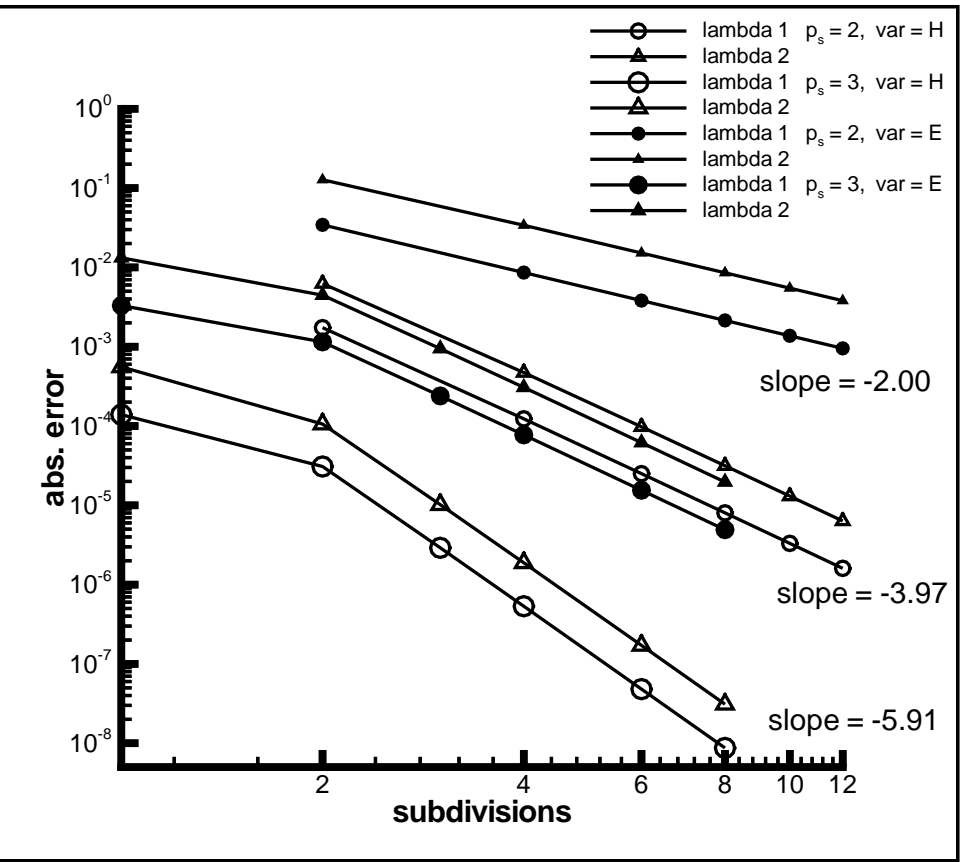

FiguRE 2. Dielectric rectangle: $h$-refinement by triangles 


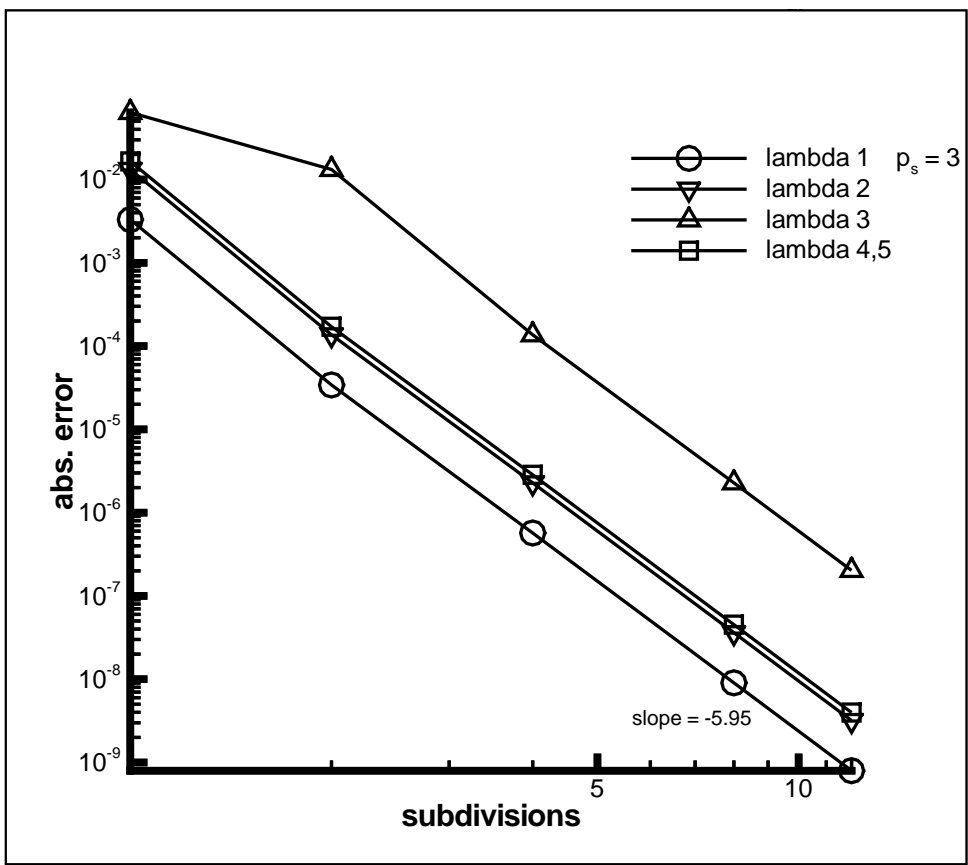

Figure 3 . Conducting rectangle: $h$-refinement by quads

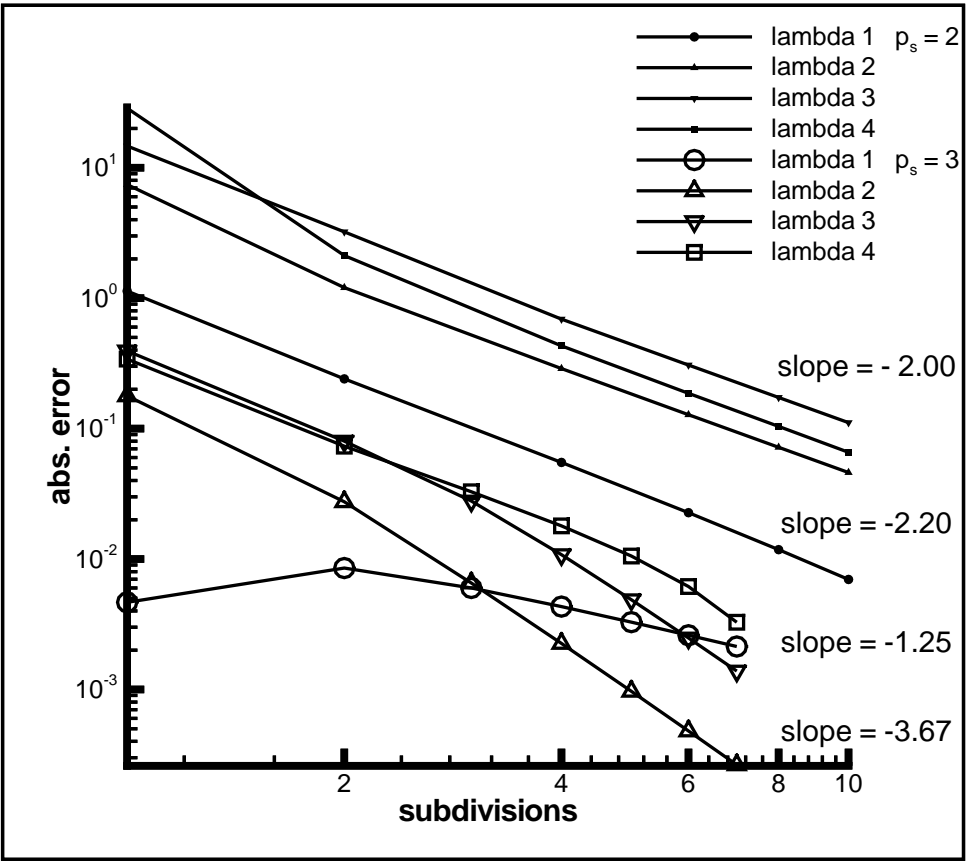

FiguRE 4. Dielectric sector: $h$-refinement by isoparametric triangles 
For the plots in Figures 1 and 2 the cross section $\Omega$ is taken as a $1 \times 2$ rectangle at $k=0.2$ with $\sigma=0$. The slopes are in full agreement with (6.42).

In Figure 2, the eigenvalues $\lambda_{1}$ and $\lambda_{2}$ correspond to the TE-modes. This implies that $\boldsymbol{\nabla} \times \boldsymbol{H}=0$. Therefore, as predicted by theory and now seen in the plots, solving Maxwell's equations for $\left(\boldsymbol{H}, H_{3}\right)$, not for $\left(\boldsymbol{E}, E_{3}\right)$, provides for higher rates of convergence.

For the plots in Figure 3 , the cross section $\Omega$ is taken as a $1 \mathrm{~mm} \times 2 \mathrm{~mm}$ rectangle at $100 \mathrm{KHz}$ with $\epsilon_{r}=1, \mu_{r}=1, \sigma=5.8 \cdot 10^{4} \mathrm{~S} / \mathrm{mm}$. Although the theoretical results apply only to nonlossy waveguides, the rates of convergence are as expected.

For an example of domains with singular eigenmodes, we take the cross section $\Omega$ as a $3 / 2 \pi$-sector of unit radius. The eigenmode with the lowest regularity of $r=2 / 3-\delta, \delta>0$, corresponds to $\lambda_{1}$, the smallest eigenvalue. Since $\Omega$ is not a polygon, a map isoparametric with respect to the scalar element is used to model curved elements. The rates of convergence given in Figure 4 are as expected in all plots but one: for $\lambda_{1}$ the rate is higher then expected if $p_{s}=2$.

\section{ApPendix: On CONVERGENCE ESTIMATES}

We note that the results of [6] cannot be directly applied to the problem at hand because we did not demonstrate compactness of $\boldsymbol{T}$ on the space $\boldsymbol{X}$, where the isomorphism $L$ of (4.5) corresponding to the form $\boldsymbol{a}_{\omega}$ is defined.

Let us simplify our notation and denote the pairs $\left(\boldsymbol{E}, E_{3}\right)$ and $(\boldsymbol{F}, q)$ we have been working with by the single letters $u$ and $v$. Thus, in this simplified notation (4.33) and (4.34) take on the form

$$
\begin{gathered}
\boldsymbol{a}_{\omega}(\hat{\boldsymbol{T}} u, v)=(u, v)_{\mathcal{H}}, \quad \forall u \in \mathcal{H}, \quad \forall v \in \boldsymbol{X}, \\
\boldsymbol{a}_{\omega}\left(u, \hat{\boldsymbol{T}}^{*} v\right)=(u, v)_{\mathcal{H}}, \quad \forall u \in \boldsymbol{X}, \quad \forall v \in \mathcal{H} .
\end{gathered}
$$

Similarly, (4.38) with $\boldsymbol{T}$ and (4.42) with $\boldsymbol{T}_{*}$ become

$$
\begin{array}{ll}
\boldsymbol{a}_{\omega}(\boldsymbol{T} u, v)=(\mathbb{B} u, v)_{\mathcal{H}}, & \forall u \in \mathcal{H}, \quad \forall v \in \boldsymbol{X}, \\
\boldsymbol{a}_{\omega}\left(u, \boldsymbol{T}_{*} v\right)=(\mathbb{B} u, v)_{\mathcal{H}}, & \forall u \in \boldsymbol{X}, \quad \forall v \in \mathcal{H} .
\end{array}
$$

We recall that the convergence estimate of Theorem 7 involves $\boldsymbol{T}$ and its adjoint $\boldsymbol{T}^{*}$, not the operator $\boldsymbol{T}_{*}$. Next, we investigate how the spectral properties of $\boldsymbol{T}^{*}$ relate to those of $\boldsymbol{T}_{*}$. As a result of Theorem 3 we can define a bounded operator $A: \boldsymbol{X} \rightarrow \boldsymbol{X}$ by

$$
\boldsymbol{a}_{\omega}(u, v)=[A u, v]_{\boldsymbol{X}}, \quad \forall u \in \boldsymbol{X}, \quad \forall v \in \boldsymbol{X},
$$

where $[\cdot, \cdot]_{\boldsymbol{X}}$ is the inner product on $\boldsymbol{X}$. The adjoint of $A$ with respect to this inner product, denoted by $A^{\prime}$, can be defined by

$$
\boldsymbol{a}_{\omega}(u, v)=\left[u, A^{\prime} v\right]_{\boldsymbol{X}}, \quad \forall u \in \boldsymbol{X}, \quad \forall v \in \boldsymbol{X} .
$$

Theorem 3 also implies that both $A^{-1}$ and $\left(A^{\prime}\right)^{-1}$ exist as bounded operators on $\boldsymbol{X}$.

Now, we note that the $[\cdot, \cdot]_{\boldsymbol{X}}$-inner product, as a sesquilinear form on $\boldsymbol{X}$, satisfies the "inf - sup" conditions. Therefore, we can define a bounded operator $M: \mathcal{H} \longrightarrow$ $\boldsymbol{X}$ as

$$
[u, M v]_{\boldsymbol{X}}=(u, v)_{\mathcal{H}}, \quad \forall u \in \boldsymbol{X}, \quad \forall v \in \mathcal{H}
$$


By the definition of $A^{\prime}$ in (A.6) and by the identity $A^{\prime}\left(A^{\prime}\right)^{-1}=I d$ on $\boldsymbol{X}$, we can write (A.7) as

$$
(u, v)_{\mathcal{H}}=[u, M v]_{X}=\left[u, A^{\prime}\left(A^{\prime}\right)^{-1} M v\right]_{X}=\boldsymbol{a}_{\omega}\left(u,\left(A^{\prime}\right)^{-1} M v\right)
$$

to get the following identity:

$$
(u, v)_{\mathcal{H}}=\boldsymbol{a}_{\omega}\left(u,\left(A^{\prime}\right)^{-1} M v\right), \quad \forall u \in \boldsymbol{X}, \forall v \in \mathcal{H} .
$$

Let us show now that

$$
\begin{array}{rll}
\sigma\left(\boldsymbol{T}^{*}\right) & \subset & \sigma\left(\boldsymbol{T}_{*}\right), \\
\left(A^{\prime}\right)^{-1} M \operatorname{Ker}\left(\boldsymbol{T}^{*}-\bar{\lambda}\right)^{j} & \subset & \operatorname{Ker}\left(\boldsymbol{T}_{*}-\bar{\lambda}\right)^{j}, \quad \forall \bar{\lambda} \in \sigma\left(\boldsymbol{T}^{*}\right) .
\end{array}
$$

We take any $\bar{\lambda} \in \sigma\left(\boldsymbol{T}^{*}\right)$ and any generalized eigenvector $v^{*}$ associated with $\bar{\lambda}$ so that $v^{*} \in \operatorname{Ker}\left(\boldsymbol{T}^{*}-\bar{\lambda}\right)^{j}$ :

$$
\left(\boldsymbol{T}^{*}-\bar{\lambda}\right)^{j} v^{*}=0
$$

and obviously

$$
\left(u,\left(\boldsymbol{T}^{*}-\bar{\lambda}\right)^{j} v^{*}\right)_{\mathcal{H}}=0, \quad \forall u \in \boldsymbol{X} .
$$

Since $\boldsymbol{T}^{*}$ is the $\mathcal{H}$-adjoint of $\boldsymbol{T}$, A.12) yields

$$
\left((\boldsymbol{T}-\lambda)^{j} u, v^{*}\right)_{\mathcal{H}}=0, \quad \forall u \in \boldsymbol{X} .
$$

Now, by (A.9), (A.13) implies that

$$
\boldsymbol{a}_{\omega}\left((\boldsymbol{T}-\lambda)^{j} u,\left(A^{\prime}\right)^{-1} M v^{*}\right)=0, \quad \forall u \in \boldsymbol{X} .
$$

Using the identity (which can be verified by induction to hold on $\boldsymbol{X} \times \boldsymbol{X}$ )

$$
\boldsymbol{a}_{\omega}\left((\boldsymbol{T}-\lambda)^{s} y, z\right)=\boldsymbol{a}_{\omega}\left(y,\left(\boldsymbol{T}_{*}-\bar{\lambda}\right)^{s} z\right), \quad s=0,1,2, \ldots,
$$

we can conclude that (A.14) yields

$$
\boldsymbol{a}_{\omega}\left(u,\left(\boldsymbol{T}_{*}-\bar{\lambda}\right)^{j}\left(A^{\prime}\right)^{-1} M v^{*}\right)=0, \quad \forall u \in \boldsymbol{X} .
$$

Since $\boldsymbol{a}_{\omega}$ satisfies the "inf - sup" conditions on $\boldsymbol{X}$, it follows from (A.16) and (A.11) that

$$
\left(\boldsymbol{T}_{*}-\bar{\lambda}\right)^{j}\left(A^{\prime}\right)^{-1} M v^{*}=0, \quad \forall v^{*} \in \operatorname{Ker}\left(\boldsymbol{T}^{*}-\bar{\lambda}\right)^{j} .
$$

Thus, (A.17) confirms both inclusions in A.10.

Let $\lambda$ be a nonzero eigenvalue of (4.4) with algebraic multiplicity $m$. This also means that $\lambda^{-1}$ is an eigenvalue of $\boldsymbol{T}$, with equal multiplicity.

Let us use the result (6.38) of Theorem 7 to bound $\left|\lambda^{-1}-\lambda_{i, h}^{-1}\right|^{\kappa}$ by $\varepsilon_{h}$ and $\varepsilon_{h}^{*}$ introduced in (6.36) and (6.37).

We consider $\left\|\left.\left(\boldsymbol{T}-\boldsymbol{T}_{h}\right)\right|_{R(\boldsymbol{E})}\right\|_{\mathcal{L}(\mathcal{H}, \mathcal{H})}$ first. From 4] and [5] and (4.2), (4.29), (4.30), (4.3), (4.38), (5.9), (5.13), and (5.16) we have, for any $u \in \mathcal{H}$,

$$
\left\|\left(\boldsymbol{T}-\boldsymbol{T}_{h}\right) u\right\|_{\mathcal{H}} \leq\left\|\left(\boldsymbol{T}-\boldsymbol{T}_{h}\right) u\right\|_{\boldsymbol{X}} \leq\left(1+\frac{C}{\alpha_{0}}\right) \inf _{\chi_{h} \in \boldsymbol{X}_{h}}\left\|\boldsymbol{T} u-\chi_{h}\right\|_{\boldsymbol{X}} .
$$

Recalling that the range of the spectral projection $R(\boldsymbol{E})$ is invariant with respect to $\boldsymbol{T}$ (see e.g., [6]), for any $u \in R(\boldsymbol{E})$ we obtain

$$
\inf _{\chi_{h} \in \boldsymbol{X}_{h}}\left\|\boldsymbol{T} u-\chi_{h}\right\|_{\boldsymbol{X}}=\inf _{\chi_{h} \in \boldsymbol{X}_{h}}\left\|\frac{\boldsymbol{T} u}{\|\boldsymbol{T} u\|_{\mathcal{H}}}-\chi_{h}\right\|_{\boldsymbol{X}}\|\boldsymbol{T} u\|_{\mathcal{H}} \leq \varepsilon_{h}\|\boldsymbol{T}\|_{\mathcal{L}(H, H)}\|u\|_{\mathcal{H}}
$$


Therefore, combining (A.18) and (A.19) and using the definition of operator norm, we get

$$
\left\|\left.\left(\boldsymbol{T}-\boldsymbol{T}_{h}\right)\right|_{R(\boldsymbol{E})}\right\|_{\mathcal{L}(\mathcal{H}, \mathcal{H})}=\sup _{u \in S(\lambda)}\left\|\left(\boldsymbol{T}-\boldsymbol{T}_{h}\right) u\right\| \leq \frac{C}{\alpha_{0}} \varepsilon_{h},
$$

where $C$ is some positive constant independent of $h$.

For any $u \in \mathcal{H}$ with $\|u\|_{\mathcal{H}}=1$ and for any $v^{*} \in R\left(\boldsymbol{E}^{*}\right)$ with $\left\|v^{*}\right\|_{\mathcal{H}}=1$, we have by A.9

$$
\begin{aligned}
((\boldsymbol{T} & \left.\left.-\boldsymbol{T}_{h}\right) u, v^{*}\right)_{\mathcal{H}}=\boldsymbol{a}_{\omega}\left(\left(\boldsymbol{T}-\boldsymbol{T}_{h}\right) u,\left(A^{\prime}\right)^{-1} M v^{*}\right) \\
& =\boldsymbol{a}_{\omega}\left(\left(\boldsymbol{T}-\boldsymbol{T}_{h}\right) u,\left(A^{\prime}\right)^{-1} M v^{*}-\chi_{h}\right) \\
& \leq C\left\|\left(\boldsymbol{T}-\boldsymbol{T}_{h}\right) u\right\|_{\boldsymbol{X}}\left\|\left(A^{\prime}\right)^{-1} M v^{*}-\chi_{h}\right\|_{\boldsymbol{X}}, \quad \forall \chi_{h} \in \boldsymbol{X}_{h} .
\end{aligned}
$$

In (A.21), the appearance of any $\chi_{h} \in \boldsymbol{X}_{h}$ is justified by (4.38), (5.13) and (5.16).

Let us demonstrate that $\left(A^{\prime}\right)^{-1} M$ is a bounded operator on $\mathcal{H}$. Indeed, since $\left(A^{\prime}\right)^{-1} \in \mathcal{L}(\boldsymbol{X}, \boldsymbol{X})$, and $M: \mathcal{H} \longrightarrow \boldsymbol{X}$ is bounded, the following chain of inequalities holds for any $u \in \mathcal{H}$ :

$$
\begin{aligned}
\left\|\left(A^{\prime}\right)^{-1} M u\right\|_{\mathcal{H}} \leq\left\|\left(A^{\prime}\right)^{-1} M u\right\|_{\boldsymbol{X}} & \leq\left\|\left(A^{\prime}\right)^{-1}\right\|_{\mathcal{L}(\boldsymbol{X}, \boldsymbol{X})}\|M u\|_{\boldsymbol{X}} \\
& \leq\left\|\left(A^{\prime}\right)^{-1}\right\|_{\mathcal{L}(\boldsymbol{X}, \boldsymbol{X})}\|M\|_{\mathcal{L}(\mathcal{H}, \boldsymbol{X})}\|u\|_{\mathcal{H}} .
\end{aligned}
$$

Therefore,

$$
\left\|\left(A^{\prime}\right)^{-1} M\right\|_{\mathcal{L}(\mathcal{H}, \mathcal{H})} \leq\left\|\left(A^{\prime}\right)^{-1}\right\|_{\mathcal{L}(\boldsymbol{X}, \boldsymbol{X})}\|M\|_{\mathcal{L}(\mathcal{H}, \boldsymbol{X})} .
$$

We know from (A.17) that $\left(A^{\prime}\right)^{-1} M$ maps

$$
R\left(\boldsymbol{E}^{*}\right)=\operatorname{Ker}\left(\left(\bar{\lambda}^{-1}-T^{*}\right)^{\kappa}\right) \quad \text { into } \operatorname{Ker}\left(\left(\bar{\lambda}^{-1}-T_{*}\right)^{\kappa}\right),
$$

with $\kappa$ being the ascent for $\bar{\lambda}^{-1}$. Therefore, just like in (A.19) we can obtain

$$
\inf _{\chi_{h} \in \boldsymbol{X}_{h}}\left\|\left(A^{\prime}\right)^{-1} M v^{*}-\chi_{h}\right\|_{\boldsymbol{X}} \leq \varepsilon_{h}^{*}\left\|\left(A^{\prime}\right)^{-1} M\right\|_{\mathcal{L}(H, H)}\left\|v^{*}\right\|_{\mathcal{H}} .
$$

Consequently, we get for (A.21)

$$
\left|\left(\left(\boldsymbol{T}-\boldsymbol{T}_{h}\right) u, v^{*}\right)_{\mathcal{H}}\right| \leq C\left\|\left(\boldsymbol{T}-\boldsymbol{T}_{h}\right) u\right\|_{\boldsymbol{X}}\left\|\left(A^{\prime}\right)^{-1} M\right\|_{\mathcal{L}(H, H)} \varepsilon_{h}^{*}
$$

where $C$ is a positive constant. From (A.25) it is immediate that, for any $v^{*} \in$ $R\left(\boldsymbol{E}^{*}\right),\left\|v^{*}\right\|_{\mathcal{H}}=1$

$$
\begin{aligned}
\left\|\left(\boldsymbol{T}^{*}-\boldsymbol{T}_{h}^{*}\right) v^{*}\right\|_{\mathcal{H}} & =\sup _{u \in \mathcal{H}} \frac{\left|\left(u,\left(\boldsymbol{T}^{*}-\boldsymbol{T}_{h}^{*}\right) v^{*}\right)_{\mathcal{H}}\right|}{\|u\|_{\mathcal{H}}} \\
& \leq C\left\|\boldsymbol{T}-\boldsymbol{T}_{h}\right\|_{\mathcal{L}(\mathcal{H}, \boldsymbol{X})}\left\|\left(A^{\prime}\right)^{-1} M\right\|_{\mathcal{L}(H, H)} \varepsilon_{h}^{*} .
\end{aligned}
$$

We recall that $\lim _{h \rightarrow 0}\left\|\boldsymbol{T}_{h}-\boldsymbol{T}\right\|_{\mathcal{L}(H, H)}=0$. This implies that $\left\|\boldsymbol{T}-\boldsymbol{T}_{h}\right\|_{\mathcal{L}(\mathcal{H}, \boldsymbol{X})}$ is uniformly bounded with respect to $h$. Thus, we can obtain from (A.26)

$$
\left\|\left.\left(\boldsymbol{T}^{*}-\boldsymbol{T}_{h}^{*}\right)\right|_{R\left(\boldsymbol{E}^{*}\right)}\right\|_{\mathcal{L}(H, H)} \leq \frac{C}{\alpha_{0}} \varepsilon_{h}^{*},
$$

where $C$ is some positive constant. By using (A.25), A.18) and A.19 we may bound $\left|\left(\left(\boldsymbol{T}-\boldsymbol{T}_{h}\right) \phi_{i}, \phi_{j}^{*}\right)\right|$, where $\phi_{i} \in R(\boldsymbol{E}), \phi_{i}^{*} \in R\left(\boldsymbol{E}^{*}\right)$, and $\left\|\phi_{i}\right\|_{\mathcal{H}}=\left\|\phi_{j}^{*}\right\|_{\mathcal{H}}=1$, 
as follows:

$$
\begin{aligned}
\left|\left(\left(\boldsymbol{T}-\boldsymbol{T}_{h}\right) \phi_{i}, \phi_{j}^{*}\right)_{\mathcal{H}}\right| & \leq C_{1}\left\|\left(\boldsymbol{T}-\boldsymbol{T}_{h}\right) \phi_{i}\right\|_{\boldsymbol{X}}\left\|\left(A^{\prime}\right)^{-1} M\right\|_{\mathcal{L}(H, H)} \varepsilon_{h}^{*} \\
& \leq \frac{C_{2}}{\alpha_{0}}\|\boldsymbol{T}\|_{\mathcal{L}(H, H)}\left\|\left(A^{\prime}\right)^{-1} M\right\|_{\mathcal{L}(H, H)} \varepsilon_{h} \varepsilon_{h}^{*},
\end{aligned}
$$

where $C_{1}, C_{2}$ are some positive constants.

Estimates (A.20), A.27) and A.28 indicate that indeed

$$
\begin{aligned}
& \sum_{j, l=1}^{m}\left|\left(\left(\boldsymbol{T}-\boldsymbol{T}_{h}\right) \phi_{j}, \phi_{l}^{\prime}\right)_{\mathcal{H}}\right| \\
& \quad+\left\|\left.\left(\boldsymbol{T}-\boldsymbol{T}_{h}\right)\right|_{R(\boldsymbol{E})}\right\|_{\mathcal{L}(\mathcal{H}, \mathcal{H})}\left\|\left.\left(\boldsymbol{T}^{*}-\boldsymbol{T}_{h}^{*}\right)\right|_{R\left(\boldsymbol{E}^{*}\right)}\right\|_{\mathcal{L}(\mathcal{H}, \mathcal{H})} \leq \frac{C}{\alpha_{0}} \varepsilon_{h} \varepsilon_{h}^{*} .
\end{aligned}
$$

Remark 4. Theorems 8.1-8.4 dealing with the convergence estimates for eigenvalues and eigenvectors stated in [6] by Babuška and Osborn are all valid for our case due to the estimates (A.20), A.27) and A.28.

Acknowledgments. The authors would like to thank Professor Babuška, Professor Boffi, and Professor Monk for insightful and invigorating discussions on the subject.

\section{REFERENCES}

[1] N. I. Akhiezer and I. M. Glazman, Theory of linear operators in Hilbert space, Dover, NY, 1993. MR 94i: 47001

[2] A. Alonso and A. Valli, "An Optimal Domain Decomposition Preconditioner for LowFrequency Time-Harmonic Maxwell Equations", Math. Comp., 68, 226, 607-631, April 1999. MR 99i:78002

[3] D. N. Arnold, R. S. Falk, and R. Winther, "Multigrid in H(div) and H(curl)", Numer. Anal., 85, 197-217, 2000. MR 2001d:65161

[4] I. Babuška, "Error Bounds for Finite Element Method", Numer. Math., 16, 322-333, 1971. MR 44:6166

[5] I. Babuška and A. Aziz, "Survey Lectures on the Mathematical Foundations of the Finite Element Method", in A. K. Aziz, ed., The Mathematical Foundations of the Finite Element Method with Application to Partial Differential Equations, 5-359, Academic Press, NY, 1973, pp. 1-359. MR 54:9111]

[6] I. Babuška and J. Osborn, Eigenvalue Problems, in Handbook of Numerical Analysis, vol.2, Elsevier-North Holland, Amsterdam, 1991, pp. 641-787. MR 92f:65001

[7] A. Bermúdez and D. G. Pedreira, "Mathematical Analysis of a Finite Element Method without Spurious Solutions for Computation of Dielectric Waveguides", Numer. Math., 61, 39-57, 1992. MR 92m:65139

[8] D. Boffi, "A note on the de Rham complex and a discrete compactness property", Appl. Math. Lett. 14, 33-38, 2001. MR 2001g:65145

[9] _ , "Fortin operator and discrete compactness for edge elements", Numer. Math. 87, 229-246, 2000. CMP 2001:06

[10] F. Chatelin Spectral Approximations of Linear Operators, Academic Press, NY, 1983. MR 86d:65071

[11] L. Demkowicz, "Asymptotic Convergence in Finite and Boundary Element Methods: Part 1: Theoretical Results", Comput. Math. Appl., 27, 12, 69-84, 1994. MR 95h:65080

[12] L. Demkowicz, P. Monk, L. Vardapetyan, and W. Rachowicz, "De Rham Diagram for $h p$ Finite Element Spaces", Comput. Math. Appl., 39, no. 7/8, 29-38, 2000. MR 2000m:78052

[13] L. Demkowicz, P. Monk, Ch. Schwab, and L. Vardapetyan, "Maxwell Eigenvalues and Discrete Compactness in Two Dimensions", Comput. Math. Appl., 40, no. 4/5, 589-605, 2000. CMP 2000:16

[14] L. Demkowicz, L. Vardapetyan, "Modeling of Electromagnetic Absoption/Scattering Problems Using $h p$-adaptive Finite Elements", Comput. Methods Appl. Mech. Engrg., 152, 1-2, 103-124, 1998. MR 99b:78003 
[15] S. Caorsi, P. Fernandes, M. Raffetto, "On the Convergence of Galerkin Finite Element Approximations of Electromagnetic Eigenproblems", SIAM J. Numer. Anal., 38, 580-607, 2000. MR 2001e:65172

[16] V. Girault and P. A. Raviart, Finite Element Methods for Navier-Stokes Equations, SpringerVerlag, Berlin, 1986. MR 88b:65129

[17] P. Joly, C. Poirier, J. E. Roberts, and P. Trouve, "A New Nonconforming Finite Element Method for the Computation of Electromagnetic Guided Waves I: Mathematical Analysis", SIAM J. Numer. Anal., 33 4, 1494-1525, 1996. MR 97f:78035

[18] F. Kikuchi, "Mixed and Penalty Formulations for Finite Element Analysis of an Eigenvalue Problem in Electromagnetism", Comput. Methods Appl. Mech. Engrg., 64, 509-521, 1987. MR 89g:78005

[19] F. Kikuchi, "On a Discrete Compactness Property for the Nedelec Finite Elements", J. Fac. Sci., Univ. Tokyo, Sect. IA Math, 36, 479-490, 1989. MR 91h:65173

[20] F. Kikuchi, "Discrete Compactness of the Linear Rectangular Nedelec Element", Abstracts of Presentations at 1999 Spring Meeting of Math. Soc. Japan, 108-111, 1999.

[21] F. Kikuchi, M. Yamamoto, H. Fujio, "Theoretical and Computational Aspects of Nedelec's Edge Elements for Electromagnetics", in Computational Mechanics - New Trends and Applications, Eds.:E. Oñate and S. R. Idelsohn, CIMNE, Barcelona, Spain, 1998.

[22] J.-F. Lee, "Finite Element Analysis of Lossy Dielectric Waveguides", IEEE Transactions on Microwave Theory and Techniques, MTT-42, 1025-1031, 1994

[23] J.F. Lee, D.K. Sun, and Z.J. Cendes, "Full-Wave Analysis of Dielectric Waveguides Using Tangential Vector Finite Elements", IEEE Transactions on Microwave Theory and Techniques, 39, 8, 1991.

[24] R. Leis, Initial Boundary Value Problems in Mathematical Physics, John Wiley and Sons, New York 1986. MR 87h:35003

[25] P. Monk and L. Demkowicz, "Discrete Compactness and the Approximation of Maxwell's Equations in $\mathbb{R}^{3} "$, Math. Comp., 70, 507-523, 2001. MR 2001g:65156

[26] J.C. Nedelec, "Mixed Finite Elements in $\mathbb{R}^{3 ",}$ Numer. Math., 35, 315-341, 1980. MR 81k:65125

[27] J.C. Nedelec, "A New Family of Mixed Finite Elements in $\mathbb{R}^{3 "}$, Numer. Math., 50, 57-81, 1986. MR 88e: 65145

[28] J.T. Oden and L.F. Demkowicz, Applied Functional Analysis for Science and Engineering, CRC Press, Boca Raton, 1996. MR 97h:00001

[29] J. E. Osborn, "Spectral Approximation for Compact Operators", Math. Comp., 29, 131, 712-725, 1975. MR 52:3998

[30] W. Rachowicz and L. Demkowicz, "A Two-Dimensional $h p$-Adaptive Finite Element Package for Electromagnetics", TICAM Report 98-15, July 1998, accepted, Comput. Methods Appl. Mech. Engrg..

[31] P. P. Silvester and G. Pelosi (eds.), Finite Elements for Wave Electromagnetics, IEEE Press, NY, 1994.

[32] L. Vardapetyan, hp-Adaptive Finite Element Method for Electromagnetics with Applications to Waveguiding Structures, Ph.D. thesis, Graduate School of The University of Texas at Austin, December 1999.

[33] L. Vardapetyan and L. Demkowicz, "hp-Adaptive Finite Elements in Electromagnetics", Comput. Methods Appl. Mech. Engrg., 169, 331-344, 1999. MR 99k:78004

[34] C. Weber, "A Local Compactness Theorem for Maxwell's Equations", Math. Meth. Appl Sci., 2, 12-25, 1980. MR 81f:78005

The Texas Institute for Computational and Applied Mathematics, The University of Texas at Austin, Taylor Hall 2.400, Austin, Texas 78712

E-mail address: leonv@research.bell-labs.com

The Texas Institute for Computational and Applied Mathematics, The University of Texas at Austin, Taylor Hall 2.400, Austin, Texas 78712

E-mail address: leszek@ticam.utexas.edu 